\title{
Über die oxydative und die vermeintliche synthetische Bildung von Harnsäure in Rinderleberauszug.
}

\author{
Von
}

Richard Burian.

Mit acht Kurvenzeichnungen.

(Aus dem physiologischen Institut der Universität Leipzig.)

(Der Redaktion zugegangen am 11. Januar 1905.)

Durch die im Anschluß an die grundlegenden Horbaczewskischen Experimente ${ }^{1}$ ) ausgeführten schönen Untersuchungen von Spitzer ${ }^{2}$ ) und von Wiener ${ }^{3}$ ) ist mit voller Sicherheit erwiesen, daß die Auszüge verschiedener Säugetierorgane, insbesondere die der Leber und der Milz, eine spezifische 0xydase enthalten, die bei Körpertemperatur und Gegenwart von Sauerstoff Hypoxanthin und Xanthin zu Harnsäure zu oxydieren vermag, und die ich deshalb im nachfolgenden als "Xanthinoxydase * bezeichnen will. Auch Adenin und Guanin werden, wie Schittenhelm ${ }^{4}$ ) gezeigt hat, durch die oxydasehaltigen Organextrakte in Harnsäure umgewandelt, jedoch erst nachdem sie zuvor durch ein Desamidierungsferment der Auszüge (die Guanase von Jones und Partridge) ${ }^{5}$ ) in Hypoxanthin resp. Xanthin übergeführt worden sind. Nucleinsäuren und Nucleoproteide erleiden in den Extrakten zunächst

1) Monatsh. f. Chem., Bd. 10, S. 624, und Bd. 12, S. 221.

2) Pflügers Arch. f. d. ges. Physiol., Bd. 76, S. 192.

$\left.{ }^{3}\right)$ Arch. f. exp. Pathol. u. Pharmakol., Bd. 42, S. 373 ; vgl. besonders Tabelle IX, Versuch 38, S. 397.

4) Diese Zeitschrift, Bd. XLII, S. 251, und Bd. XLIII, S. 228.

5) Diese Zeitschrift, Bd. XLII, S. 343. Vgl. übrigens auch Burian und Walker Hall, Diese Zeitschrift, Bd. XXXVIII, S. 372 u. 382, und Burian, Ergebnisse der Physiologie, Bd. 3. I., S. 85, wo bereits darauf hingewiesen ist, daß beim längeren Stehen von Organauszügen in der Wärme die Aminopurine derselben zu Oxypurinen desamidiert werden. 
eine Zersetzung unter Abspaltung der Purinbasen: diese letzteren unterliegen dann (eventuell nach vorausgehender Desamidierung) gleichfalls der Oxydation zu Harnsäure (Schittenhelm).

Ich habe nun die Einwirkung der Xanthinoxydase des Rinderleberauszuges auf zugesetztes Xanthin messend verfolgt. Für eine derartige Untersuchung sind die gewöhnlichen, in der Wärme hergestellten Extrakte nicht zu verwenden, weil in ihnen stets erhebliche Quantitäten von Purinbasen und zwar wechselnde Gemische verschiedener Basen - zugegen sind, und weil überdies aus den in den Auszügen enthaltenen Nucleoproteiden während der Digestion noch weitere Purinbasenmengen abgespalten werden, sodaß die Festsetzung der in Rechnung zu ziehenden Konzentrationen ganz unmöglich ist. Man wird diesem Übelstande heute am besten dadurch abhelfen, daß man die Xanthinoxydase zunächst mit Ammonsulfat aussalzt, wodurch sie nach Schittenhelms bedeutsamer Entdeckung von den Nucleoproteiden und Purinbasen der Auszüge getrennt werden kann. Bei meinen Versuchen ist von diesem Verfahren noch nicht Gebrauch gemacht, da sie ber eits einige Zeit vor Schittenhelms einschlägigen Veröffentlichungen ausgeführt wurden. Ich habe vielmehr ein anderes einfaches Mittel zur Umgehung der erwähnten Schwierigkeit benützt, das wenigstens bei Rinderleber vollkommen zu dem gewünschtenZiele führte.

Wie mich eine zufällige Beobachtung lehrte, kann man nämlich bei Anwendung dauernder guter Eiskühlung aus Rinderleber mittels Chloroformwasser (1 Gewichtsteil Leberbrei +2 Gewichtsteile Chloroformwasser) unschwer Auszüge bereiten, ${ }^{1}$ ) die nur sehr geringe Quantitäten von Purinbasen - höchstens den 3. bis 4. Teil der in gewöhnlichen Extrakten vorhandenen Menge - und nur Spuren von Nucleoproteiden, dagegen gut wirksame Xanthinoxydase enthalten. Solche purinbasenarme «aktive» Auszüge liefern ohne Xanthinkörperzusatz bei Digestion unter $\mathrm{O}_{2}$-Zufuhr

1) Dauerndes Schütteln oder Rühren des Leberbreies mit dem zur Extraktion verwendeten Chloroformwasser ist durchaus überflüssig. - Die übrigen Details der Herstellung ziemlich zellfreier Auszüge siehe bei Spitzer, l. c. 196. 
so gut wie keine Harnsäure; sie haben deshalb vor den purinbasenreichen Extrakten den Vorzug, die Einwirkung der Oxydase auf zugesetztes Xanthin oder Hypoxanthin fast ganz rein hervortreten $\mathrm{zu}$ lassen und so die Geschwindigkeitsmessung $\mathrm{zu}$ ermöglichen. Zur Durchführung der letzteren ist es natürlich notwendig, nicht bloß, wie dies die früheren Untersucher getan haben, die aus der zugesetzten Purinbasis entstandene Harnsäure, sondern auch den jeweils übrig gebliebenen Rest der Purinbasis selbst zu bestimmen. - Außer zur Geschwindigkeitsmessung eignen sich die purinbasenarmen Auszüge, wie wir sehen werden, ganz besonders zur Entscheidung der Frage, ob neben der oxydativen auch noch eine synthetische Harnsäurebildung in den Rinderleberextrakten vor sich gehen kann.

\section{Methodik.}

In den unten mitgeteilten Versuchen erfolgte die Digestion der Extrakte in einem auf $40^{\circ}$ eingestellten Thermostaten mit. Chlorcalciumregulator. Da es für die Zwecke der Geschwindigkeitsmessung ganz besonders wesentlich erschien, die Wirkung von Mikroorganismen auszuschließen, durch welche die gebildete Harnsäure partiell wieder zersetzt werden könnte, ${ }^{1}$ ) so wurden die mit Chloroformwasser bereiteten Auszüge vor der Digestion noch einmal mit einer reichlichen Menge Chloroform versetzt. Die Proben, bei denen Sauerstoffabschluß beabsichtigt war, blieben, mit einer dicken Ölschichte bedeckt, in verschlossenen Flaschen im Thermostaten stehen. Durch die Proben dagegen, bei denen Sauers toffzufuhr stattfinden sollte, wurde aus einer Sauerstoffbombe während der ganzen Dauer der Digestion ein kräftiger Sauerstoffstrom hindurchgeleitet, sodaß die Flüssigkeit stets mit Sauerstoff gesättigt bleiben mußte. Diese Maßnahme ist bei den Geschwindigkeitsmessungen unerläßlich, damit die Oxydase während der Digestion sicher ununterbrochen zu voller Wirkung gelangen könne. - Die zu den Extrakten hinzugefügten Purinstoffe waren jedesmal durch die N-Bestimmung auf ihre Reinheit geprüft.

1) Kreidl, Monatsh. f. Chem., Bd. 14, S. 109. 
Nach Beendigung der Digestion wurden die Auszüge behufs Unterbrechung der Reaktion und Vorbereitung für die Bestimmungen - in das mehrfache Volumen siedender Kochsalzlösung eingegossen ${ }^{1}$ ) und unter Zusatz von etwas Essigsäure koaguliert. Sowohl in der Flüssigkeit als auch im Koagulum wurde der Purinkörpergehalt ermittelt.

Die Flüssigkeit wurde stark eingeengt, sodann mit etwas Natronlauge (aus Natrium bereitetes Natriumhydrat!) aufgekocht und rasch filtriert. In dem mit Salzsäure angesäuerten, erkalteten Filtrat wurde die Harnsäure nach dem (zum ersten Male von v. Schröder ${ }^{2}$ ) auf Organextrakte angewandten) LudwigSalkowskischen Verfahren bestimmt. Die abgeschiedene "Rohharnsäure», die übrigens meist ohne weiteres in den für reinste Harnsäure charakteristischen hexagonalen Täfelchen auskristallisierte, wurde zur endgültigen Reinigung nach dem Vorgange von Horbaczewski ${ }^{3}$ ) aus konzentrierter Schwefelsäure (2ccm pro $0,1 \mathrm{~g}$ Rohharnsäure) mit dem vierfachen Volumen Wasser umgefällt. - Zur Ermittelung des Purinbasen-N in den vereinigten Mutterlaugen der Roh- und Reinharnsäure diente die von mir an anderer Stelle ${ }^{4}$ ) beschriebene Modifikation der Salkowskischen Methode.

Das Koagulum wurde behufs Prüfung auf etwa eingeschlossene Harnsäure und Purinbasen in viel verdünnter, siedender Natronlauge (aus Natrium bereitetes Natriumhydrat!) gelöst, die Flüssigkeit sodann in der Siedehitze mit Essigsäure schwach angesäuert und von den hierdurch neuerlich ausgefällten Eiweißsubstanzen durch einen Heißwassertrichter abfiltriert. Das Filtrat wurde nach den oben genannten Methoden auf Purinstoffe verarbeitet. Indessen ließen sich nur bei jenen Proben, zu welchen die Purinsubstanzen in besonders reichlicher Menge (Tabelle III, Extrakt I, Probe F), oder aber in ungelöster Form (Tabelle I, Extrakt II, Proben E und F, und Tabelle II, Extrakt I, Proben

1) Daß die Wirksamkeit der Xanthinoxydase durch Aufkochen vernichtet wird, ist zuerst von Wiener, l. c. 392, nachgewiesen worden.

2) Festschrift für Carl Ludwig, S. 89.

s) Diese Zeitschrift, Bd. XVIII, S. 341.

4) Archiv f. die ges. Physiol., Bd. 80, S. 283. 
Über oxydative etc. Bildung von Harnsäure in Rinderleberauszug. 501

A-D) hinzugefügt worden waren, aus dem Koagulum noch größere oder geringere Quantitäten von Harnsäure, resp. Basen gewinnen. Bezüglich der in diesenFällen erhaltenenRohharnsäure sei bemerkt, daß sie gewöhnlich noch mit etwas eiweißartiger Substanz verunreinigt war. Zur Beseitigung derselben wurde das Produkt in warmer Ammoniakflüssigkeit aufgenommen und die abgekühlte Lösung für mehrere Stunden in Eis gestellt; das Eiweiß bleibt hierbei in Lösung, während das harnsaure Ammon quantitativ auskristallisiert. Das letztere wurde in Harnsäure übergeführt, und diese hierauf der Schwefelsäurebehandlung nach Horbaczewski unterworfen. Daß dies Verfahren für quantitative $Z$ wecke brauchbar ist, ergibt sich aus den in Tabelle II für die Proben A und B angeführten Harnsäurezahlen.

Die aus den digerierten Auszügen erhaltene Harnsäure wurde in jenen Fällen, wo ihre Menge hierzu ausreichte, der N-Bestimmung unterzogen. Es läßt sich dadurch indessen ebensowenig, wie durch die C- und H-Bestimmung, eine geringfügige Verunreinigung der Harnsäure durch Purinbasen mit Sicherheit ausschließen. ${ }^{1}$ ) Ein zuverlässiges Kriterium hierfür liefert hingegen die Untersuchung der gewonnenen Produkte mit Diazobenzolsulfosäure, weil sich dabei selbst sehr unbedeutende Purinbasenbeimengungen durch Rotfärbung verraten. ${ }^{2}$ ) Der Empfindlichkeitsgrad der Reaktion und die bei Ausführung derselben zweckmäßig einzuhaltenden Konzentrationsverhältnisse lassen sich aus nachfolgender Zusammenstellung entnehmen:

\section{Reine Harnsäure.}

$1 \mathrm{~g}$ Harnsäure, gelöst in $250 \mathrm{ccm} 8 \%$ iger Natronlauge:

Diazoreaktion negativ.

$1 \mathrm{~g}$ Harnsäure, gelöst in $50 \mathrm{ccm} 8 \%$ iger Natronlauge:

Diazoreaktion negativ.

2. Harnsäure, mit Xanthin verunreinigt.

$1 \mathrm{~g}$ Harnsäure gelöst in $50 \mathrm{ccm} 8 \%$ iger Natronlauge: $+0,02$. Xanthin Diazoreaktion negativ.

$1 \mathrm{~g}$ Harnsäure ) gelöst in $250 \mathrm{ccm} 8 \%$ iger Natronlauge : $+0,05$. Xanthin Diazoreaktion negativ.

1) Vgl. Wulff, Diese Zeitschrift, Bd. XVII, S. 639.

2) Vgl. Burian, Ber. d. Deutsch. chem. Ges., Bd. 37, S. 696. 
$1 \mathrm{~g}$ Harnsäure ) gelöst in $50 \mathrm{ccm} 8 \%$ iger Natronlauge: $+0,05$, Xanthin $\}$ Diazoreaktion deutlich positiv.

3. Harnsäure, mit Guanin verunreinigt.

$1 \mathrm{~g}$ Harnsäure ) gelöst in $50 \mathrm{ccm} 8 \%$ iger Natronlauge: $+0,02$. Guanin $\}$ Diazoreaktion schwach positiv.

$1 \mathrm{~g}$ Harnsäure gelöst in $250 \mathrm{ccm} 8 \%$ iger Natronlauge: $+0,05>$ Guanin $\}$ Diazoreaktion schwach positiv.

$1 \mathrm{~g}$ Harnsäure gelöst in $50 \mathrm{ccm} 8 \%$ iger Natronlauge: $+0,05 \gg$ Guanin Diazoreaktion intensiv.

Aus dieser Zusammenstellung ergibt sich folgende Vorschrift für die Untersuchung von Harnsäure auf etwa beigemengte Purinbasen: Man löse $0,1 \mathrm{~g}$ der Substanz in $5 \mathrm{ccm}$ heißer verdünnter Natronlauge und füge nach dem Erkalten ein wenig Diazobenzolsulfosäure hinzu: eine Verunreinigung mit nur 0,002-0,003 g Guanin oder $0,005 \mathrm{~g}$ Xanthin, welche sich in den $\mathrm{N}$-, C- und HWerten gar nicht ausprägen könnte, würde sich dabei bereits durch deutliche Rotfärbung zu erkennen geben.

Auf solche Art geprüft, erwies sich die aus den digerierten Auszügen gewonnene Harnsäure in allen Fällen als frei von jeglicher Purinbasenbeimengung.

Es bietet sich hier Gelegenheit, einer irrigen Ansicht entgegenzutreten, die H. Pauly kürzlich in einer aus Kossels Laboratorium stammenden Arbeit ${ }^{1}$ ) bezüglich der Diazoreaktion geäußert hat. Pauly stellt es als zweifelhaft hin, ob die Diazokörperderivate der Imidazole wirklich Diazoaminoverbindungen seien. «Mir scheint, so schreibt er, ein Ansehung ihrer Beständigkeit in saurer Lösung die Möglichkeit nicht ausgeschlossen, daß in ihnen, ähnlich wie beim Pyrrol, echte Azokörper . . . . . vorliegen.s

Hierzu bemerke ich, daß allerdings vor meiner diesbezüglichen Veröffentlichung meines Erachtens keine Berechtigung vorhanden war, das bis dahin allein bekannte Diazobenzolimidazol mit Sicherheit als Diazoaminoverbindung anzusprechen. Eben deshalb habe ich aber die verschiedenartigsten Substitutionsprodukte des Imidazols in den Kreis der Untersuchung gezogen: es ergab sich hierbei, daß das $\mu$-Methylund das $\mu$-Phenylimidazol, das $\mu$-Sulf hydryl- $\alpha$-, $\beta$-Diphenylimidazol, die Imidazol- $\alpha$-, $\beta$-Dikarbonsäure, die $\mu$-Methyl- und die $\mu$-Phenylimidazol$\alpha, \beta$-Dikarbonsäure, kurz sämtliche untersuchten $\alpha-, \beta$ - und $\mu$-Substitutionsprodukte des Imidazols mit Diazokörpern zu

1) Diese Zeitschrift, Bd. XLII, S. 508. 
Über oxydative etc. Bildung von Harnsäure in Rinderleberauszug. 503<smiles></smiles>

reagieren vermögen, und zwar auch dann, wenn alle drei genannten Stellen gleichzeitig besetzt sind, während die Substitution des Imidwasserstoffatoms im n-Methylimidazol sofort die Reaktionsfähigkeit a ufhebt. Lassen diese Tatsachen eine andere Deutung zu als die, daß der Diazorest bei der Reaktion an das Stickstoffatom $\mathbf{n}$ herantritt?

Ich möchte ferner betonen, daß ich niemals empfohlen habe, «die Diazoreaktion etwa allgemein zur Unterscheidung des Imidazolringes vom Pyrimidinring zu verwertens, wovor Pauly warnt, und daß ich die Diazoreaktion auch bei den Purinbasen keineswegs a priori auf deren Imidazolring bezog. Erst nachdem ich mich durch die Untersuchung des Einflusses, den Substitutionen auf die Reaktionsfähigkeit der Purinbasen ausüben, davon überzeugt hatte, daß die Anlagerung des Diazorestes an den Purinkern bei Stelle 7 erfolgt, ${ }^{1}$ ) habe ich die Diazoreaktion der Purinbasen auf den Imidazolring derselben zurückgeführt.

\section{Versuchsergebnisse.}

Es seien zunächst - der Kürze halber tabellarisch einige Resultate mitgeteilt, welche den Unterschied im Verhalten der purinbasenarmen Auszüge einerseits und der gewöhnlichen, purinbasenreichen Extrakte anderseits illustrieren (vgl. die umstehende Tab. I).

Die Ergebnisse der Tabelle I lassen sich in folgende Sätze zusammenfassen:

1. Bei Digestion unter Sauerstoffabschluß findet sowohl in purinbasenarmen, wie in purinbasenreichen Leberauszügen, ja selbst bei Purinbasenzusatz, keine merkliche Harnsäurebildung statt (siehe Extrakt II, Probe B und C, Kolumne a, Extrakt I, Probe B und D, Kolumne a). Zugesetzte Purinbasen werden unter diesen Bedingungen unverändert quantitativ wiedergewonnen (vergleiche bei Extrakt I, Probe D, Kol. c mit Probe B, Kol. c).

1) Vgl. diesbezüglich meine Arbeit, Ber. d. Deutsch. chem. Gesellschaft, Bd. 37, S. 696, und meine Zurückweisung der Steudelschen Kritik, Diese Zeitschrift, Bd. XLII, S. 297. 
Tabelle I.

\begin{tabular}{|c|c|c|c|c|c|c|c|c|}
\hline Extrakt & Probe & Zusatz & $\begin{array}{c}\mathrm{O}_{2^{-}} \\
\text {Durch- } \\
\text { leitung }\end{array}$ & $\begin{array}{c}\text { Di- } \\
\text { gestions- } \\
\text { dauer } \\
\text { in } \\
\text { Stunden }\end{array}$ & \begin{tabular}{|c} 
a \\
Harn- \\
säure \\
mg
\end{tabular} & \begin{tabular}{|c}
$\mathbf{b}$ \\
Harn- \\
säure- \\
$\mathbf{N}$ \\
$\mathbf{m g}$
\end{tabular} & $\begin{array}{c}\text { c } \\
\text { Purin- } \\
\text { basen- } \\
\text { N } \\
\text { mg }\end{array}$ & $\begin{array}{c}\text { d } \\
\text { Gesamt- } \\
\text { purin-N } \\
\text { mg }\end{array}$ \\
\hline \multirow{4}{*}{$\begin{array}{c}\text { I. } \\
\text { Gewöhnlicher } \\
\text { purin- } \\
\text { basen- } \\
\text { reicher } \\
\text { Rinderleber- } \\
\left.\text { auszug, }{ }^{1}\right) \\
\text { für jede Probe } \\
1500 \mathrm{ccm} \\
\text { entsprechend } \\
750 \text { g Leber- } \\
\text { brei }\end{array}$} & A & $\varnothing$ & - & $\varnothing$ & 5,1 & 1,7 & 104,4 & 106,1 \\
\hline & B & $\varnothing$ & - & 10 & 4,5 & 1,5 & 157,2 & 158,7 \\
\hline & G & $\varnothing$ & + & 10 & 162,0 & 54,0 & 1,5 & 55,5 \\
\hline & D & $\begin{array}{l}\text { Je } 180 \mathrm{mg} \\
\text { Hypo- } \\
\text { x anthin } \\
\text { (in Subst.) } \\
\text { entspr. } \\
74,7 \text { mg N }\end{array}$ & + & 10 & 230,7 & 76,9 & 227,2 & 230,3 \\
\hline \multirow{4}{*}{$\begin{array}{c}\text { II. } \\
\text { Purin- } \\
\text { basen- } \\
\text { armer } \\
\text { Rinderleber- } \\
\text { auszug, }\end{array}$} & A & $\varnothing$ & - & $\varnothing$ & 1,2 & 0,4 & 24,1 & 24,5 \\
\hline & B & $\varnothing$ & - & 5 & 2,0 & 0,7 & 26,9 & 27,6 \\
\hline & C & $\varnothing$ & - & 10 & 1,5 & 0,5 & 28,7 & 29,2 \\
\hline & D & ø & + & 5 & 9,2 & 3,1 & Spurer & 3,1 \\
\hline \multirow{3}{*}{$\begin{array}{l}\text { für jede Probe } \\
1000 \mathrm{ccm} \\
\text { entsprechend } \\
500 \mathrm{~g} \mathrm{Leber-} \\
\text { brei }\end{array}$} & $\mathrm{E}$ & $\varnothing$ & + & 10 & 5,5 & 1,8 & $\varnothing$ & 1,8 \\
\hline & F & $\begin{array}{c}\mathrm{Je} 600 \mathrm{mg} \\
\mathrm{X} \text { anth in } \\
\text { (in Sub- }\end{array}$ & + & 5 & 157,7 & 52,6 & 133,8 & 186,4 \\
\hline & G & $\begin{array}{l}\text { stanz) ent- } \\
\text { sprechend } \\
221 \mathrm{mg} \mathrm{N}\end{array}$ & + & 10 & 90,4 & 30,1 & 100,4 & 130,5 \\
\hline
\end{tabular}

Bemerkung: Die bei Probe C und E des Extraktes I erhatenen Harnsäuren wurden vereinigt und zur N-Bestimmung verwendt. 0,1309 g Substanz: $31,0 \mathrm{ccm} \mathrm{1/10} \mathrm{norm.} \mathrm{Säure,} \mathrm{entsprechend} \mathrm{0,0434} \mathrm{g} \mathrm{N} \mathrm{:}$ gefunden $\mathbf{3 3 , 1 5} \%$, berechnet $\mathbf{3 3 , 3 3} \% \mathrm{~N}$.

1) Dieser Extrakt war nicht unter Anwendung von Kälte hergestellt worden. 
Über oxydative etc. Bildung von Harnsẩure in Rinderleberauszug. 505

2. Bei Digestion unter Sauerst of fzufuhr bilden purinbasenreiche Leberauszüge sehr erhebliche, purinbasenarme dagegen kaum nennenswerte Harnsäuremengen (siehe einerseits Extrakt I, Probe C, Kol. a, andererseits Extrakt II, Probe D und E, Kol. a). Bei Purinbasenzusatz jedoch ist die Harnsäurebildung unter diesen Umständen nicht bloß in den purinbasenreichen Extrakten gesteigert (vergleiche bei Extrakt I, Probe E, Kol. a mit Probe G, Kol. a), sondern es liefern dann auch die ursprünglich purinbasenarmen Auszüge ansehnliche Harnsäuremengen (vergleiche bei Extrakt II, Probe F, Kol. a mit Probe D, Kol. a; ferner Probe G, Kol. a mit Probe E, Kol. a).

Es ist demnach sowohl die Gegenwart von Purinbasen, wie auch die von Sauerstoff unbedingt erforderlich, wenn eine Harnsäurebildung in den Leberextrakten vor sich gehen soll.

3. Bemerkenswert ist das Verhalten der Purinbasen: dieselben nehmen bei Digestion unter Sauerstofflüftung - also in jenen Extrakten, in denen sich eine Harnsäurebildung vollzieht, - stets beträchtlich ab (vergl. z. B. bei Extrakt I, Kol. c, die Probe $\mathrm{C}$ resp. E mit B resp. D); der Verlust an Basen-N ist indessen größer, als der Zuwachs an Harnsäure-N. Dieser letztere Umstand, der natürlich ein Absinken des Gesamtpurin-N (Kol. d) bewirkt, könnte zweierlei Ursache haben: entweder erfolgt die Purinbasenzersetzung teilweise durch eine Reaktion, die mit der Harnsäurebildung nichts zu tun hat, oder aber die aus den Purinbasen entstandene Harnsāure unterliegt selbst wieder einer weiteren Zerstörung.

In der Tat zeigen nun die Auszüge von Rinderleber bei Gegenwart von Sauerstoff außer dem Vermögen der Harnsäurebildung - freilich in geringerem Grade - auch die Fähigkeit, zugesetzte Harnsäure zu zerstören. Am übersichtlichsten liegen die Verhältnisse wieder bei purinbasenarmen Extrakten, in welchen die Abnahme der zugesetzten Harnsäure, nicht durch eine spontane Harnsäurebildung verdeckt, ohne weiteres zu Tage treten kann. Fügt man zu solchen Extrakten Harnsäure in Substanz hinzu, so erleidet dieselbe 
bei Digestion unter Sauerstoffdurchleitung allerdings nur eine recht geringfügige Verminderung. ${ }^{1}$ ) Man darf jedoch nicht außer acht lassen, daß die in ungelöstem Zustande zugesetzte schwerlösliche Harnsäure erst während der Digestion allmählich in Lösung gehen muß, wobei sicher niemals völlige Sättigung erreicht wird. - Gelangt die Harnsäure, in (wenig) Alkali gelöst, zur Verwendung, so nimmt sie bei der Digestion des Auszuges sehr beträchtlich ab. Es ist freilich zu berücksichtigen, daß in alkalischen Harnsäurelösungen bei $\mathrm{O}_{2}$-Zufuhr auch ohne Gegenwart von Leberextrakt eine oxydative Zerstörung der Harnsäure statthat $;^{2}$ ) indessen ist diese Zerstörung doch ganz erheblich weniger umfänglich als bei Anwesenheit von Leberextrakt. Zweifellos besitzt also die Rinderleber die Fähigkeit, Harnsäure zu zersetzen, wenngleich in sehr viel schwächerem Grade als Hundeleber. ${ }^{3}$ )

Belege für das Gesagte erbringt Tabelle II.

Für die Proben E-K dieser Tabelle wurden die abgewogenen Harnsäuremengen in je $20 \mathrm{ccm} \mathrm{1/2-n} \mathrm{Na}_{2} \mathrm{CO}_{3}$-Lösung unter Kochen aufgelöst, hierauf je $20 \mathrm{ccm}$ siedenden Wassers hinzugefügt, sodann die mäßig abgekühlten Lösungen mit $360 \mathrm{ccm}$ Rinderleberauszug $(\mathrm{E}-\mathrm{G})$ oder Chloroformwasser $(\mathrm{H}-\mathrm{K})$ auf $400 \mathrm{ccm}$ aufgefüllt. Die digerierten Lösungen besaßen somit in allen Fällen annähernd die gleiche schwache Alkaleszenz.

Die (spontane) Harnsäurebildung ist in den für die Proben A-F angewandten purinbasenarmen Auszügen - nach den in Tab. I, Extrakt II, bei Probe $D$ und $E$ und in Tab. III, Extrakt II, bei Probe D, E und F angegebenen Harnsäurewerten - so gering zu veranschlagen, daß sie den großen zugesetzten Harnsäuremengen gegenüber vollständig vernachlässigt werden kann.

1) Bei Digestion unter Sauerstoffabschluß ist eine Abnahme der Harnsäure überhaupt nicht zu konstatieren (vergl. Tab. II, Probe A u. B).

2) Staedeler, Liebigs Ann., Bd. 78, S. 286. - Nencki u. Sieber, Journ. f. prakt. Chem., Bd. 24, S. 503. - v. Schröder, l. c., S. 94. Kreidl, Monatsh. f. Chem., Bd. 14, S. 114.

3) Daß das Harnsäurezerstörungsvermögen der Hundeleber (und ebenso der Katzen- und Schweineleber) ein außerordentlich großes ist, wurde bekanntlich durch zahlreiche Untersuchungen festgestellt. Vgl. z. B. Chassevant u. Richet, Compt. rend. de la Soc. de biol., X., Bd. 4, S. 743. Jacoby, Virchows Arch., Bd. 157, S. 261. Wi ener, Arch. f. experim. Pathol. u. Pharm., Bd. 42, S. 357. Ascoli, Pflügers Arch., Bd. 72, S. 340. Burian u. Schur, Pflügers Arch., Bd. 87, S. 306-318, etc. 
Über oxydative etc. Bildung von Harnșäure in Rinderleberauszug. 507

Tabelle II.

\begin{tabular}{|c|c|c|c|c|c|c|c|c|c|}
\hline Extrakt & Roaktion & Probo & Zusatz & $\begin{array}{c}0_{2}- \\
\text { Darch- } \\
\text { leithong }\end{array}$ & $\begin{array}{l}\text { Di- } \\
\text { gestions- } \\
\text { dauer } \\
\text { in } \\
\text { Stunden }\end{array}$ & $\begin{array}{c}\mathbf{a} \\
\text { Harn- } \\
\text { säure } \\
\mathbf{m g}\end{array}$ & \begin{tabular}{|c|}
$\mathbf{b}$ \\
Harn- \\
säure- \\
$\mathrm{N}$ \\
$\mathbf{m g}$
\end{tabular} & $\begin{array}{c}\text { c } \\
\text { Purin- } \\
\text { basen- } \\
\mathrm{N} \\
\mathbf{m g}\end{array}$ & $\begin{array}{c}\text { d } \\
\text { Gesamt- } \\
\text { purin-N } \\
\text { mg }\end{array}$ \\
\hline $\begin{array}{c}\quad \text { I. } \\
\text { Der in Tab. I } \\
\text { als Extrakt II } \\
\text { bezeichnete } \\
\text { purin- } \\
\text { basen- }\end{array}$ & \multirow{2}{*}{$\begin{array}{l}\text { Neu- } \\
\text { tral }\end{array}$} & B & \multirow{2}{*}{$\begin{array}{c}\text { Je } 600 \mathrm{ccm} \\
\text { Harnsäure } \\
\text { (entsprechend } \\
200 \mathrm{mg} \mathrm{N} \text { ) } \\
\text { inSubstanz }\end{array}$} & - & .5 & $\begin{array}{l}595,3 \\
591,9\end{array}$ & $\begin{array}{l}198,4 \\
197,3\end{array}$ & $\begin{array}{l}29,9 \\
28,1\end{array}$ & $\begin{array}{l}228,3 \\
225,4\end{array}$ \\
\hline $\begin{array}{c}\text { arme } \\
\text { Auszug, } \\
\text { für jedeProbe } \\
\text { verwendet } \\
1000 \mathrm{ccm} \\
\text { Extrakt }\end{array}$ & & $D$ & & $\begin{array}{l}+ \\
+\end{array}$ & 5 & $\begin{array}{l}-519,0 \\
500,2\end{array}$ & $\begin{array}{l}173,0 \\
166,7\end{array}$ & $\begin{array}{l}2,6 \\
1,8\end{array}$ & $\begin{array}{l}175,6 \\
168,5\end{array}$ \\
\hline \multirow{6}{*}{$\begin{array}{c}\text { II. } \\
\text { Der in Tab. III } \\
\text { als Extrakt II } \\
\text { bezeichnete } \\
\text { purin- } \\
\text { basen- } \\
\text { arme } \\
\text { Extrakt, } \\
\text { für jede Probe } \\
\text { verwendet } \\
360 \mathrm{ccm}\end{array}$} & \multirow{3}{*}{$\begin{array}{l}\text { Sethrach } \\
\text { alkalisch }\end{array}$} & $\mathrm{E}$ & $\begin{array}{c}500 \mathrm{mg} \text { Harn- } \\
\text { säure (entsprech. } \\
166,6 \mathrm{mg} \mathrm{N}) \text { in } \\
\mathrm{Na}_{2} \mathrm{CO}_{3} \mathrm{~g} \text { elö st }\end{array}$ & + & 5 & $184 ; 0$ & $\ddot{61,3}$ & 4,8 & 66,1 \\
\hline & & $\mathrm{F}$ & \multirow{2}{*}{$\begin{array}{l}200 \mathrm{mg} \text { Harn- } \\
\text { säure } \\
\text { (entsprechend } \\
66,6 \mathrm{mg} \mathrm{N} \text { ) } \\
\text { in } \mathrm{Na}_{2} \mathrm{CO}_{3} \\
\text { gelöst }\end{array}$} & + & 5 & 73,7 & 24,6 & 6,5 & 31,1 \\
\hline & & G & & + & 8 & 45,0 & 15,0 & 6,6 & 21,6 \\
\hline & \multirow{3}{*}{$\begin{array}{l}\text { Sehroach } \\
\text { allaliech }\end{array}$} & $\mathrm{H}$ & $\begin{array}{l}531 \mathrm{mg} \text { Harn- } \\
\text { säure in } \mathrm{Na}_{2} \mathrm{CO} \mathrm{O}_{3} \\
\text { gelöst, auff } \\
400 \mathrm{ccm} \text { aufgefüllt }\end{array}$ & + & 4 & 499,0 & 166,3 & - & - \\
\hline & & I & $\begin{array}{c}450 \mathrm{mg} \text { Harn- } \\
\text { säure in } \mathrm{Na}_{2} \mathrm{CCO}_{3} \\
\text { gelöst, auf } \\
400 \mathrm{ccm} \text { aufgefüllt }\end{array}$ & + & 7 & 400,7 & 133,6 & - & - \\
\hline & & $\mathrm{K}$ & $\begin{array}{c}200 \mathrm{mg} \text { Harm- } \\
\text { säure in Na} \mathrm{CO}_{3} \\
\text { gelöst, auf } \\
400 \mathrm{ccm} \text { aufgefüllt }\end{array}$ & + & 8 & 167,8 & 55,9 & - & - \\
\hline
\end{tabular}


Die Beobachtung, daß Rinderleberauszug Harnsäure zu zersetzen imstande ist, steht in Widerspruch zu einer Angabe von Jacoby, ${ }^{1}$ ) nach welcher Kalbs- und Rinderleber - im Gegensatz zu Hundeleber -- kein Harnsäurezerstörungsvermögen aufweisen soll. Diese Angabe erklärt sich daraus, daß Jacoby die das Harnsäuredefizit verdeckende spontane Harnsäurebildung nicht berücksichtigte, welche in seinen zwei Experimenten eine nicht $\mathrm{zu}$ unterschätzende Rolle gespielt haben dürfte. ${ }^{2}$ ) In Anbetracht des letzteren Umstandes darf man, da Jacoby die zugesetzte Harnsäure nur eben quantitativ $(95-97 \%$ ) wiederfand, sogar mit ziemlicher Sicherheit schließen, daß auch in diesen Versuchen eine geringgradige Harnsäurezersetzung stattgefunden hat.

Mit der Tatsache des Harnsäurezerstörungsvermögens der Rinderleber steht im Einklang, daß zu Rinderleberauszug hinzugefügte Purinbasen bei der Digestion mit $\mathrm{O}_{\mathbf{2}}$ bisher niemals quantitativ als Harnsäure wiedergewonnen wurden (vgl. z. B. Spitzer, Pflügers Archiv, Bd. 76, S. 198, Vers. 7, Schittenhelm, Diese Zeitschrift, Bd. XLII, S. 258, Vers. 8), während bei Extrakten aus Kalbsmilz, denen die Befähigung zur Harnsäurezerstörung abgeht, bereits mehrmals vollständige Überführung der zugesetzten Purinbasen in Harnsäure beobachtet worden ist (z. B. Spitzer, l. c. 197, Vers. 6, Schittenhelm, l. c. 256, Vers. 1).

Nach dem vorstehenden liegt es nahe, anzunehmen, daß die bei der Digestion von Rinderleberextrakt mit Sauerstoff regelmäßig zu konstatierende Gesamtpurinabnahme lediglich durch die Zerstörung der aus den Purinbasen entstehenden Harnsäure bedingt ist, daß die Purinbasen selbst aber ausschließlich in Harnsäure übergehen und keinerlei andersartige Umwandlung erleiden. Wirklich werden nun die Folgerungen, die sich aus dieser Annahme für den zeitlichen Verlauf des Prozesses ableiten lassen, durch die Erfahrung vollauf bestätigt. Am einfachsten gestaltet sich die Untersuchung bei jener Basis, die der Harnsäure bereits am nächsten steht,

1) Virchows Archiv, Bd. 157, S. 261.

2) Jacoby arbeitete mit einer Suspension von Leberbrei in Chloroformwasser, in der die spontane Harnsäurebildung all ermin de s tens ebenso groß sein muß, wie in den gewöhnlichen purinbasenreichen Leberextrakten. 
Über oxydative etc. Bildung von Harnsäure in Rinderleberauszug. 509

beim Xanthin. ${ }^{1}$ ) Hier gelingt es, bei Verwendung möglichst purinbasenarmer Auszüge unschwer festzustellen:

1. Daß das allmähliche Verschwinden des Xanthins während der Digestion einzig und allein durch Überführung desselben in Harnsäure zustande kommt; und

2. daß die gesamte im Extrakte gebildete Harnsäure so gut wie ausschließlich aus der Oxydation des Xanthins herstammt.

Unter der Voraussetzung, daß diese beiden Bedingung en erfüllt sind, und unter der Annahme, daß die Menge des wirksamen Sauerstoffes - d. h. bei genügender Lüftung also die Oxydasenmenge - während der Digestion unverändert bleibt, gelten nämlich ${ }^{2}$ ) für die Oxydation des Xanthins einerseits und für die Zersetzung der hierbei gebildeten Harnsäure andererseits, da $1 \mathrm{Mol}$. Xanthin $1 \mathrm{Mol}$. Harnsäure liefert, die Geschwindigkeitsgleichungen

$$
\begin{aligned}
& \frac{d x}{d t}=k_{1}(a-x) \ldots \ldots . . .(1) \\
& \frac{d y}{d t}=k_{2}(b+x-y) \ldots \ldots(2)
\end{aligned}
$$

worin

$$
\begin{aligned}
& a=\text { mol. Anfangskonzentration } \\
& x=\text { zur Zeit } t \text { (in } 100 \mathrm{ccm} \text { ) umgewandelte mol. Xanthinmenge } \\
& \begin{aligned}
\mathbf{b} & =\text { mol. Anfangskonzentration } \\
\mathbf{b}+\mathbf{x}-\mathbf{y} & =\text { zur Zeit } \mathbf{t} \text { bestehende mol. Konzentration } \\
\mathrm{y} & =\text { zur Zeit } \mathbf{t} \text { (in } 100 \mathrm{ccm} \text { ) zersetzte mol. Harnsauremenge. }
\end{aligned}
\end{aligned}
$$

Es ist $\mathrm{zu}$ bemerken, daß in $\mathrm{k}_{1}$ die Konzentration der Xanthinoxydase enthalten ist; da dieselbe je nach dem $\mathrm{Zu}$ stande der verwendeten Leber und nach der Bereitungsweise des Extraktes wechseln kann, so ist klar, daß $k_{1}$ nur für ein

1) Beim Hypoxanthin und Guanin und mehr noch beim Adenin würde sich Rechnung und Messung naturgemäß erheblich komplizierter gestalten.

2) Falls keine Anomalie vorliegt, sondern die gewöhnlichen Reaktionsgleichungen erster Ordnung zutreffen - eine Prämisse, deren Richtigkeit durch die unten mitgeteilten Messungsergebnisse bewiesen wird.

Hoppe-Seyler's Zeitschrift f. physiol. Chemie. XIIII. 
und denselben Auszug eine Konstante sein muß, für verschiedene Auszüge aber eine verschiedene Größe besitzen kann.

Es ergibt nun die Integration von (1)

$$
k_{1}=\frac{1}{t} \ln \frac{a}{a-x}, \ldots \ldots \ldots \ldots \text {. }
$$

die von (2) 1 )

$$
y=a+b-\frac{k_{2}}{k_{2}-k_{1}}(a-x)+C(a-x)^{\frac{k_{2}}{k_{1}}}, \ldots \ldots \text { (4) }
$$

wo $C=\frac{\frac{k_{2}}{k_{2}-k_{1}} a-(a+b)}{a^{\frac{k_{2}}{k_{1}}}}$ ist. Bei bekanntem $x$, y und $k_{1}$ läßt

sich $\mathrm{k}_{2}$ aus Gleichung (4) durch Ausprobieren ermitteln.

Ferner folgt aus den Gleichungen (1) und (2) noch, daß der Harnsäuregehalt des Auszuges während der Digestion von dem niedrigen Anfangswerte $b$ ausgehend - auf ein Maximum ansteigen muß, um sodann wieder abzusinken. Denn die Veränderung der Harnsäurekonzentration ist nach (1) und (2)

$$
\frac{d(x-y)}{d t}=k_{1}(a-x)-k_{2}(b+x-y) .
$$

Dieser Differentialquotient muß positiv sein, solange $k_{1}(a-x)>k_{2}(b+x-y)$ ist, er wird $\theta$, d. h. es tritt ein Maximum ein, wenn $k_{1}(a-x)=k_{2}(b+x-y)$, und er wird negativ, wenn $k_{1}(a-x)<k_{2}(b+x-y)$ ist. $\left.{ }^{2}\right)$

1) Die Integration der Gleichung (2) verdanke ich der Güte des Herrn Dr. Freundlich, Assistenten am Ostwaldschen Institut in Leipzig. - Vgl. übrigens auch die Abhandlung von M ellor u. Bradshaw Zeitschr. f. physikal. Chem., Bd. 48, S. 353, wo das Integrationsresultat für einen Spezialfall, nämlich für $b=0$, bereits angegeben ist.

2) Übrigens lehrt schon eine einfache Überlegung, daß bei der Digestion einer Purinbasis mit Rinderleberextrakt anfangs, solange die Konzentration der Base verhältnismäßig groß ist, mehr Harnsäure durch Oxydation entstehen als verschwinden wird, während später, wenn der Basengehalt relativ klein geworden ist, mehr Harnsäure zerstört als neugebildet werden muß. 
Über oxydative etc. Bildung von Harnsäure in Rinderleberauszug. 511

Die zeitliche Lage und die Höhe des Harnsäuremaximums läßt sich bei bekanntem $k_{1}$ und $k_{2}$ leicht berechnen. Tritt das Maximum zur Zeit $\tau$ ein, und ist $a-x_{\tau}$ die Xanthin-, $b+x_{\tau}-y_{\tau}$ die Harnsäurekonzentration des Auszuges zur Zeit $\tau$, so ergibt sich aus der Bedingung $k_{1}\left(a-x_{\tau}\right)$ $=\mathrm{k}_{\mathrm{z}}\left(\mathrm{b}+\mathrm{x}_{\tau}-\mathrm{y}_{\tau}\right)$ und aus der Gleichung (4):

$$
a-x_{\tau}=\left(\frac{C}{C^{\prime}}\right)^{\frac{k_{1}}{k_{1}-k_{2}}}, \ldots \ldots \ldots \ldots(5)
$$

worin $C^{\prime}=\frac{k_{1}^{2}}{k_{2}^{2}-k_{1} k_{8}}$ ist (der Wert von $G$ ist bereits bei Gleichung (4) angeführt). Da nach der Maximumsbedingung die Harnsäurekonzentration $b+x_{\tau}-y_{\tau}=\frac{k_{1}}{k_{q}}\left(a-x_{\tau}\right)$ und nach Gleichung (1) die Zeit $\tau=\frac{1}{k_{1}} \ln \frac{a}{a-x_{\tau}}$, so ist Höhe und Zeitpunkt des Harnsäuremaximums durch Gleichung (5) vollständig bestimmt.

Die hier entwickelten Gleichungen werden nun durch die Ergebnisse meiner messenden Versuche durchaus bestätigt. Bei diesen Versuchen mußte das Xanthin natürlich in gelöster Form zu den (purinbasenarmen) Auszügen hinzugefügt werden. Es wurde deshalb in soviel $1 / 2-\mathrm{n} \mathrm{Na}_{2} \mathrm{CO}_{3}$-Lösung aufgelöst, daß die zu digerierenden Extrakte durch den Zusatz der Lösung in Bezug auf $\mathrm{Na}_{2} \mathrm{CO}_{3}$ 1/40-normal wurden. Die gleiche Alkaleszenz wurde selbstverständlich auch in den ohne Xanthinzusatz digerierten Vergleichsproben erzeugt.

Da die (kalt bereiteten) Rinderleberauszüge neutral reagieren, so wird durch den unvermeidlichen Alkalizusatz eine Änderung der Bedingungen gesetzt: wie wir später sehen werden (vgl. Tab. V und die zugehörige Konstantenberechnung auf S. 524), beeinflußt der Alkaleszenzgrad die Werte von $k_{1}$ und $k_{2}$ ganz deutlich. Dies ist jedoch hier zunächst gleichgültig; an dieser Stelle handelt es sich bloß darum, ob bei einem beliebigen gleichbleibenden Alkaleszenzgrade $-k_{1}$ und $k_{2}$, wie es die oben aufgestellten Gleichungen verlangen, für ein und denselben Auszug konstant sind.

Die Ergebnisse enthält 
Tabelle III.

\begin{tabular}{|c|c|c|c|c|c|c|c|c|}
\hline Extrakt & Probe & Zusatz & $\mid$\begin{tabular}{c|}
$\mathrm{O}_{2}-$ \\
Daroh- \\
loitang
\end{tabular} & $\begin{array}{l}\text { Di- } \\
\text { gestions- } \\
\text { dauer } \\
\text { in } \\
\text { Stunden }\end{array}$ & $\begin{array}{c}\mathbf{a} \\
\text { Harn- } \\
\text { säure } \\
\mathbf{m g}\end{array}$ & $\begin{array}{c}\text { b } \\
\text { Harn- } \\
\text { säure- } \\
\mathbf{N} \\
\mathbf{m g}\end{array}$ & $\begin{array}{c}\mathbf{c} \\
\text { Purin- } \\
\text { basen- } \\
\mathbf{N} \\
\mathbf{m g}\end{array}$ & $\begin{array}{c}\text { d } \\
\text { Gesamt- } \\
\text { purin-N } \\
\text { mg }\end{array}$ \\
\hline I. & $\mathbf{A}$ & $\varnothing$ & - & $\varnothing$ & 3,7 & 1,2 & 38,7 & 39,9 \\
\hline $\begin{array}{l}\text { Für jede } \\
\text { Probe } \\
950 \mathrm{ccm}\end{array}$ & $\begin{array}{l}\mathrm{B} \\
\mathrm{G}\end{array}$ & $\begin{array}{l}\varnothing \\
\varnothing\end{array}$ & - & $\begin{array}{l}4 \\
7\end{array}$ & $\begin{array}{l}2,4 \\
3,9\end{array}$ & $\begin{array}{l}0,8 \\
1,3\end{array}$ & $\begin{array}{l}38,9 \\
38,6\end{array}$ & $\begin{array}{l}39,7 \\
39,9\end{array}$ \\
\hline $\begin{array}{l}\text { Extrakt } \\
\text { (ent- } \\
\text { sprechend }\end{array}$ & $\begin{array}{l}\text { D } \\
\text { E }\end{array}$ & $\varnothing$ & $\begin{array}{l}+ \\
+\end{array}$ & $\begin{array}{l}4 \\
7\end{array}$ & Spuren & - & $\begin{array}{l}\varnothing \\
\varnothing\end{array}$ & - \\
\hline $\begin{array}{c}475 \mathrm{~g} \\
\text { Leber) } \\
+50 \mathrm{ccm}\end{array}$ & $\begin{array}{l}F \\
G\end{array}$ & $\begin{array}{l}1083 \mathrm{mg} \\
\text { Xanthin } \\
\text { (entspr. } \\
400 \mathrm{mg} \mathrm{N} \text { ) }\end{array}$ & $\begin{array}{l}+ \\
+\end{array}$ & $\begin{array}{l}4 \\
7\end{array}$ & $\begin{array}{l}724,9 \\
518,5\end{array}$ & $\begin{array}{l}241,6 \\
172,8\end{array}$ & $\begin{array}{r}33,5 \\
5,0\end{array}$ & $\begin{array}{l}275,1 \\
177,8\end{array}$ \\
\hline $\begin{array}{c}1 / 2-\mathrm{n} \\
\mathrm{Na}_{2} \mathrm{CO}_{8}- \\
\left.\text { Lösung }{ }^{1}\right)\end{array}$ & $\mathrm{H}$ & $\begin{array}{l}541,5 \mathrm{mg} \\
\text { Xanthin } \\
\text { (entspr. } \\
200 \mathrm{mg} \mathrm{N} \text { ) }\end{array}$ & $\begin{array}{l}+ \\
+\end{array}$ & $\begin{array}{l}4 \\
7\end{array}$ & $\begin{array}{l}325,2 \\
201,1\end{array}$ & 108,4 & 17,1 & $\begin{array}{r}125,5 \\
69,1\end{array}$ \\
\hline $\begin{array}{l}\text { II. } \\
\text { Für jede }\end{array}$ & A & $\varnothing$ & - & $\varnothing$ & 3,3 & 1,1 & 18,4 & 19,5 \\
\hline $\begin{array}{l}\text { Probe } \\
380 \mathrm{ccm}\end{array}$ & $\begin{array}{l}\text { B } \\
\text { C }\end{array}$ & $\varnothing$ & - & $\begin{array}{l}1 \\
6\end{array}$ & $\begin{array}{l}6,0 \\
4,2\end{array}$ & $\begin{array}{l}2,0 \\
1,4\end{array}$ & $\begin{array}{l}12,6 \\
16,4\end{array}$ & $\begin{array}{l}14,6 \\
17,8\end{array}$ \\
\hline $\begin{array}{l}\text { (ent- } \\
\text { sprechend } \\
190 \mathrm{~g} \\
\text { Leber) }\end{array}$ & $\begin{array}{l}\text { D } \\
\text { E } \\
\text { F }\end{array}$ & $\begin{array}{l}\varnothing \\
\varnothing\end{array}$ & $\begin{array}{l}+ \\
+ \\
+\end{array}$ & $\begin{array}{l}1 \\
2,5 \\
6\end{array}$ & $\begin{array}{c}3,0 \\
5,1 \\
\varnothing\end{array}$ & $\begin{array}{l}1,0 \\
1,7 \\
\varnothing\end{array}$ & $\begin{array}{c}\text { Spuren } \\
\emptyset \\
\emptyset\end{array}$ & $\begin{array}{c}1,0 \\
1,7 \\
\varnothing\end{array}$ \\
\hline $\begin{array}{c}+80 \mathrm{ccm} \\
1 / 2-\mathrm{n} \\
\mathrm{Na}_{2} \mathrm{CO}_{8^{-}} \\
\left.\text {Lösung }{ }^{1}\right)\end{array}$ & $\begin{array}{l}G \\
H \\
I\end{array}$ & $\begin{array}{c}500 \mathrm{mg} \\
\text { Xanthin } \\
\text { (entspr. } \\
184,2 \\
\text { mg N) }\end{array}$ & $\begin{array}{l}+ \\
+ \\
+\end{array}$ & $\begin{array}{l}1 \\
2,5 \\
6\end{array}$ & $\begin{array}{l}163,5 \\
247,1 \\
236,0\end{array}$ & $\begin{array}{l}54,5 \\
82,4 \\
78,7\end{array}$ & $\begin{array}{r}140,6 \\
95,0 \\
39,4\end{array}$ & $\begin{array}{l}195,1 \\
177,4 \\
118,1\end{array}$ \\
\hline
\end{tabular}

Bemerkung: 0,2031 g der bei Probe F des Extraktes I erhaltenen Harnsäure zur N-Bestimmung verwendet: $48,2 \mathrm{ccm}$ 1/10-n Säure, entsprechend 0,0675 g N: gef. : 33,24\% N. - Desgl. 0,1512 g der bei Probe $\mathrm{H}$ des Extraktes II erhaltenen Harnsäure: $36,17 \mathrm{ccm} 1 / 10-n$ Säure, entsprechend 0,05065 g N: gef.: 33,50\% N.

1) In der zugesetzten $\mathrm{Na}_{2} \mathrm{CO}_{3}$-Lösung waren bei den Proben $\mathrm{F}-\mathrm{I}$ des Extraktes I, sowie bei den Proben G-I des Extraktes II die angegebenen Xanthinmengen enthalten. 
Über oxydative etc. Bildung von Harnsäure in Rinderleberauszug. 513

Zur Ermittelung von $k_{1}$ und $k_{2}$ aus den in dieser Tabelle angeführten Zahlen ist es nicht nötig, die molaren Harnsäureund Xanthinkonzentrationen wirklich auszurechnen. Man kann vielmehr, weil $1 \mathrm{Mol}$ Harnsäure $\left(\mathrm{C}_{5} \mathrm{H}_{4} \mathrm{~N}_{4} \mathrm{O}_{3}\right)$ und 1 Mol Xanthin $\left(\mathrm{C}_{5} \mathrm{H}_{4} \mathrm{~N}_{4} \mathrm{O}_{2}\right)$ die gleiche $\mathrm{N}$-Menge besitzen, unmittelbar die absoluten Harnsäure- resp. Xanthin-N-Werte zur Rechnung benützen, da es für das Resultat gleichgültig ist, ob a, b, $\mathrm{x}$ und $\mathrm{y}$ als solche oder alle mit demselben Faktor multipliziert in die Gleichungen (3) und (4) eingesetzt werden.

Der Wert des hier in Betracht kommenden Faktors läßt sich leicht angeben. Sind in V Kubikzentimetern einer Flüssigkeit M Milligramm Harnsäure- resp. Xanthin-N vorhanden, so daß die Konzentration daran $\frac{M}{V} \cdot 100$ beträgt, so ist, da $1 \mathrm{Mol} \mathrm{Harnsäure} \mathrm{resp.} \mathrm{Xanthin} 56000 \mathrm{mg} \mathrm{N}$ enthält, die molare Harnsäure- resp. Xanthinkonzentration $m=\frac{M \cdot 100}{V \cdot 56000}$ $=\frac{\mathrm{M}}{560 \mathrm{~V}}$. Es ist. somit $560 \cdot \mathrm{V}$ der Faktor, mit welchem $\mathrm{a}, \mathrm{b}, \mathrm{x}$ und $\mathrm{y}$ multipliziert erscheinen, wenn wir statt der mol. Konzentrationen die absoluten N-Mengen (in Milligrammen) in die Rechnung einführen.

Bezüglich des für a einzusetzenden Wertes steht man vor der Wahl, den im Vergleich zum Xanthinzusatz nur geringen ursprünglichen Purinbasengehalt der Auszüge entweder ganz zu vernachlässigen, oder ihn als Xanthin in Rechnung zu bringen. Von diesen beiden - übrigens unerheblichen - Fehlern ist der zweite entschieden der kleinere. Im nachstehenden ist deshalb unter $560 \mathrm{~V} \cdot a$ stets die Summe des zugesetzten Xanthin-N und des (nach Probe A-C der beiden Extrakte) von vornherein vorhandenen Purinbasen- $\mathrm{N}$ angegeben.

Es resultiert so die umstehende Zusammenstellung.

Die Werte für $\mathbf{k}_{1}$ zeigen dieser Zusammenstellung zufolge bei Extrakt I eine befriedigende Konstanz; bei Extrakt II weisen sie allerdings einen geringfügigen Gang auf; doch handelt es sich hier nicht um eine regelmäßige Erscheinung, da bei weiter unten mitzuteilenden Experimenten, die mit dem gleichen Extrakte und unter denselben Bedingungen angestellt wurden, nichts derartiges zu beobachten ist (vgl. Tab. IV, S. 520 und die zugehörige Konstantenberechnung $\mathrm{S}$. 521). Die für $\mathrm{k}_{2}$ gefundenen Zahlen sind bei Extrakt II vollkommen konstant, bei Extrakt I fällt nur der für Probe $\mathrm{H}$ ermittelte Wert etwas aus der Reihe. 


\begin{tabular}{|c|c|c|c|c|c|c|c|c|c|}
\hline Extrakt & $\begin{array}{l}\text { Ver- } \\
\text { suchs- } \\
\text { reihe }\end{array}$ & Probe & $560 \mathrm{Va}$ & $560 \mathrm{Vb}$ & $\mid \begin{array}{l}560 \mathrm{~V} \\
(a-x)\end{array}$ & $560 \mathrm{~V} \cdot \mathrm{y}^{1}$ & $\begin{array}{c}\mathbf{t} \\
\text { (in } \\
\text { Stun- } \\
\text { den) } \\
\end{array}$ & $\mathbf{k}_{1}$ & $\mathbf{k}_{2}$ \\
\hline \multirow{4}{*}{ I } & \multirow{2}{*}{1} & $\mathbf{F}$ & \multirow{2}{*}{438,7} & \multirow{2}{*}{1,1} & 33,5 & 164,7 & 4 & 0,645 & 0,193 \\
\hline & & G & & & 5,0 & 262,0 & 7 & 0,640 & 0,180 \\
\hline & \multirow[b]{2}{*}{2} & $\mathrm{H}$ & \multirow[b]{2}{*}{238,7} & \multirow[b]{2}{*}{1,1} & 17,1 & 114,3 & 4 & 0,658 & 0,269 \\
\hline & & I & & & 2,1 & 170,7 & 7 & 0,677 & 0,198 \\
\hline \multicolumn{8}{|c|}{ Mittel: } & 0,655 & 0,220 \\
\hline \multirow{3}{*}{ II } & \multirow{3}{*}{3} & G & \multirow{3}{*}{200,0} & \multirow{3}{*}{1,5} & 140,6 & 6,4 & 1 & 0,352 & 0,210 \\
\hline & & $\mathrm{H}$ & & & 95,0 & 24,1 & 2,5 & 0,300 & 0,200 \\
\hline & & I & & & 39,4 & 83,4 & 6 & 0,270 & 0,200 \\
\hline & & & & & & & & 0,307 & 0,203 \\
\hline
\end{tabular}

Beachtung verdient, daß die Geschwindigkeitskonstante der Xanthinoxydation für Extrakt II nicht halb so groß ist, wie für Extrakt I; da im übrigen alle Versuchsbedingungen die nämlichen waren, so muß man hieraus schließen, daß Extrakt II einen geringeren Gehalt an Xanthinoxydase besaß, als Extrakt I, obzwar beide Auszüge möglichst in der gleichen Weise und mit den gleichen Mengeverhältnissen (2 Teile Chloroformwasser auf 1 Teil Leberbrei) hergestellt waren. Um so auffallender ist es, daß die Geschwindigkeit der Harnsäurezerstörung in beiden Extrakten annähernd dieselbe Gröbe zeigt. Dies weist darauf hin, daß die Harnsäurezersetzung in den Rinderleberauszügen nicht durch dasselbe Ferment bewirkt wird, durch welches die Oxydation der Purinbasen zu Harnsäure erfolgt. Weitere Resultate, die zu der nämlichen Schlußfolgerung führen, sind unten mitgeteilt.

1) $560 \mathrm{~V} \cdot \mathrm{y}$ ist der zerstörte Harnsäure-N oder, was nach unseren Voraussetzungen dasselbe ist, der verschwundene Gesamtpurin-N, und wird gefunden, indem man von dem ursprünglichen Gesamtpurin-N, $560 \mathrm{~V} \cdot(\mathrm{a}+\mathrm{b})$, den zur Zeit $\mathrm{t}$ vorhandenen Gesamtpurin-N abzieht. 
Uber oxydative etc. Bildung von Harnsäure in Rinderleberauszug. 515

Es muß hervorgehoben werden, daß der für $k_{z}$ gefundene durchschnittliche Wert derselbe ist, der sich auch aus den Harnsäurezerstörungsversuchen, speziell aus den Harnsäurezahlen der Proben E, F und G der Tabelle II ergibt. Diese Zahlen, die bei dem gleichen Auszuge und dem gleichen Alkaleszenzgrade gewonnen sind, wie die Zahlen der Proben G, H und I des Extraktes II der Tabelle III, liefern für die Geschwindigkeit der Harnsäurezersetzung die Konstanten 0,200 (E und F), resp. 0,186 (G).

Um uns von dem - für eine ganze Klasse von Reaktionen mit Folgewirkung ${ }^{1}$ ) typischen - Gesamtverlaufe des Prozesses ein durch Kurven fixierbares Bild machen zu können, wollen wir nun noch für alle drei Versuchsreihen nach Gleichung (5) Zeitpunkt und Höhe des Harnsäuremaximums berechnen. Benützen wir hierzu die oben angeführten Mittelwerte von $k_{1}$ und $k_{2}$ so erhalten wir folgende Zahlen:

\begin{tabular}{|c|c|c|c|c|}
\hline Extrakt & $\begin{array}{c}\text { Versuchs- } \\
\text { reihe }\end{array}$ & {$\left[\begin{array}{c}560 \mathrm{~V}\left(\mathbf{a}-\mathbf{x}_{\tau}\right) \\
\text { [Purinbasen-Nin mg] }\end{array}\right.$} & {$\left[\begin{array}{l}560 \mathrm{~V}\left(\mathbf{b}+\mathbf{x}_{\tau}-\mathbf{y}_{\tau}\right) \\
{[\text { Harnsäure-N in mg] }}\end{array}\right.$} & $\begin{array}{c}\tau \\
\text { in Stunden }\end{array}$ \\
\hline \multirow{2}{*}{ I } & 1 & 79,9 & 261,3 & 2,59 \\
\hline & 2 & 46,6 & 142,6 & 2,59 \\
\hline II & 3 & 58,3 & 90,4 & 3,98 \\
\hline
\end{tabular}

In Extrakt II tritt also seiner geringen Wirksamkeit halber das Harnsäuremaximum erst nach 4 Stunden ein, während es in Extrakt I bereits nach $2^{1 / 8}$ Stunden erreicht ist.

Unter Zuhilfenahme der obigen Zahlen lassen sich die Kurven der drei Versuchsreihen leicht konstruieren.

Zum Verständnisse der auf der nächsten Seite folgenden Kurvenpaare sei bemerkt, daß der zwischen Abszisse (Zeit) und unterer Kurve eingeschlossene Ordinatenabschnitt den Harnsäure-N, der zwischen den beiden Kurven eingeschlossene Ordinatenabschnitt den Purinbasen- $\mathrm{N}$ bedeutet. Die untere Kurve stellt somit die $\mathrm{Zu}$ - und Abnahme der Harnsäuremenge, die obere das durch die Harnsäurezersetzung bewirkte kontinuierliche Absinken des Gesamtpurinquantums dar. Die durch die Be-

1) Nämlich für jene Fälle, in denen $k_{1}$ und $k_{2}$ nicht allzu weit differieren, sondern derselben Größenordnung angehören. 
stimmungen gegebenen Punkte sind mit o, die durch die Berechnung des Maximums erhaltenen Punkte mit $\times$ bezeichnet. Die geringere Geschwindigkeit der Xanthinoxydation in Extrakt II (3. Versuchsreihe) drückt sich durch einen gestreckteren Verlauf der Kurven aus.
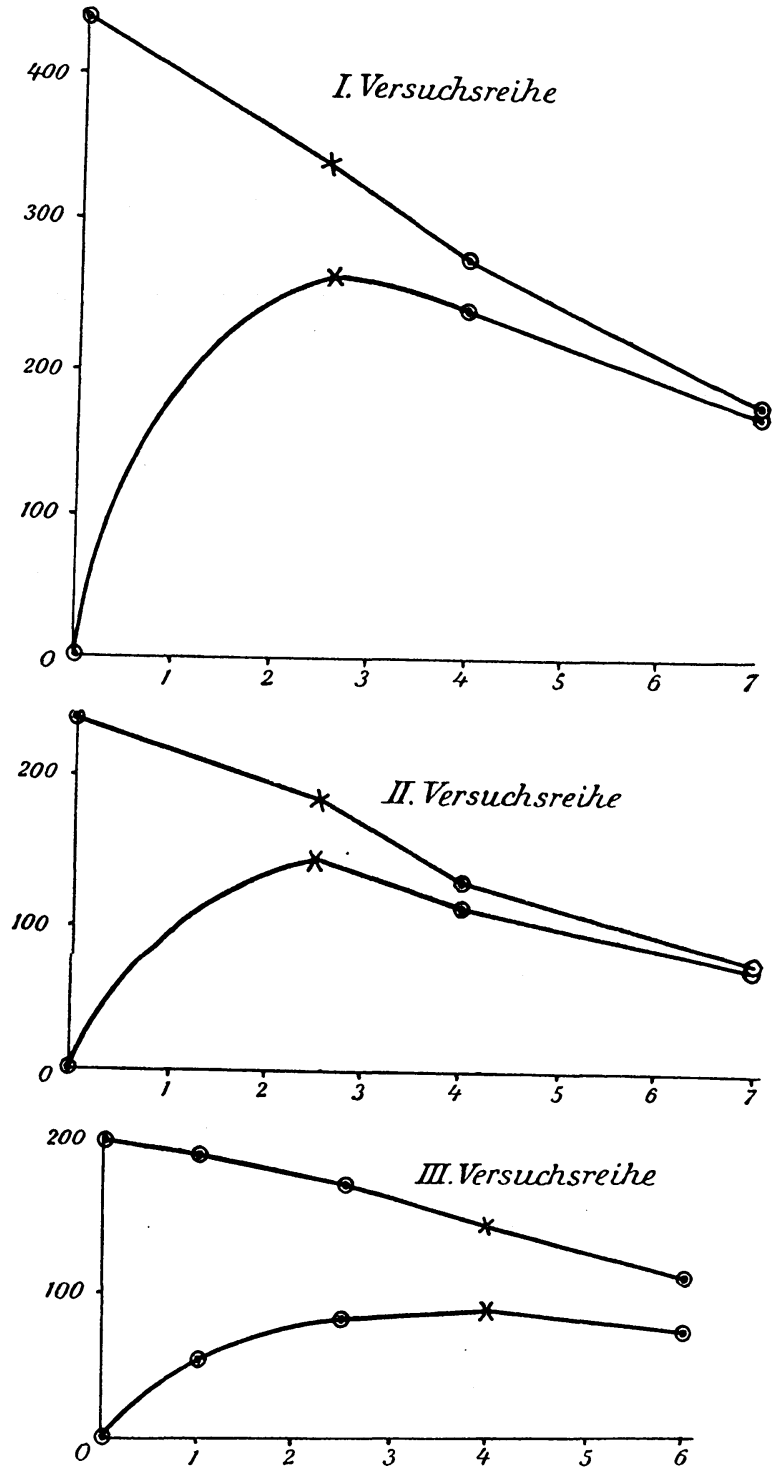
Die Übereinstimmung, die nach den mitgeteilten und den weiter unten folgenden Resultaten zwischen Versuchsergebnis. und Rechnung besteht, beweist, daß die Voraussetzungen der letzteren richtig sein müssen. Diesen Voraussetzungen zufolge stammt die im Extrakte gebildete Harnsäure so gut wie ausschließlich aus der Oxydation des Xanthins. Denn es wäre ja in Gleichung (2) die Harnsäurekonzentration $b+x-y$ unrichtig angesetzt, wenn außer der umgewandelten Xanthinmenge. $\mathrm{x}$ noch weitere Harnsäurequellen $\mathrm{zu}$ berücksichtigen wären. Dann könnte aber auch die Messung für $\mathbf{k}_{\mathbf{2}}$ unmöglich konstante Werte ergeben. Eine Harnsäurebildung durch irgend einen anderen Prozeß als den der Purinbasenoxydation, eine «synthetische» Harnsäurebildung, läßt sich also bei der Digestion von Xanthinkörpern mit purinbasenarmen Rinderleberauszügen nicht nachweisen. Hiermit steht im besten Einklange, daß die purinbasenarmen Extrakte ohne Xanthinkörperzusatz überhaupt keine nennenswerten Harnsäuremengen produzieren.

Dies besagt nun aber keineswegs, daß Rinderleberauszug die Fähigkeit zur synthetischen Harnsäurebildung gar nicht besitzt; es bleibt vielmehr noch die Möglichkeit offen, daß bloß die Ausgangsmaterialien für die Harnsäuresynthese in den Extrakten nicht oder nur in sehr geringen Mengen zugegen sind. Als solche Ausgangsmaterialien betrachtet Wien er ${ }^{1}$ ) Tartronund Dialursäure, weil die bei der Digestion purinbasenhaltiger Rinderleberauszüge vor sich gehende Harnsäurebildung durch den Zusatz dieser Substanzen merklich gesteigert wird - eine Erscheinung, die Wiener auf synthetische Entstehung von Harnsäure aus der zugesetzten Tartron- oder Dialursäure zurückführt. Ist diese Ansicht zutreffend, so ist zu erwarten, daß auch bei Digestion purinbasenarmer Extrakte mit Tartronoder Dialursäure eine deutliche Harnsäurebildung eintreten werde.

Bei Versuchen, welche ich in dieser Richtung unternommen habe, ergab sich nun ein höchst überraschendes Resultat. Digeriert man purinbasenarmen Rinderleberauszug ohne Xanthinkörperzusatz unter $\mathrm{O}_{2}$-Zufuhr mit Tartron- oder Dialursäure, so zeigt sich keine Steigerung der Harnsäure-

1) Hofmeisters Beiträge, Bd. 2, S. 42. 
bildung, die letztere ist vielmehr ebenso geringfügig, wie bei Abwesenheit der Tartron- resp. Dialursäure. Anders, wenn man den Auszug zuvor mit Xanthin versetzt hat, dann erhält man bei der Digestion mit Tartron- oder Dialursäure (falls die Versuchsdauer nicht zu groß ist) regelmäßig mehr Harnsäure, als bei der Digestion des xanthinhaltigen Auszuges allein. Mit dieser Harnsäurevermehrung ist bemerkenswerterweise stets eine Verminderung der Menge des übrig bleibenden Xanthins verbunden. Dieser Umstand spricht dafür, daß die Wirkung des Tartron- resp. Dialursäurezusatzes in einer Beschleunigung der enzymatischen Xanthinoxydation besteht; in der Tat lehrt die genauere Untersuchung, daß dem so ist. Die Geschwindigkeit der zweiten hier in Betracht kommenden Reaktion, nämlich der Harnsäurezersetzung, bleibt unverändert.

Die Experimente wurden mit dem in Tabelle III als Extrakt II bezeichneten purinbasenarmen Auszuge angestellt. Zum Vergleiche wurde eine Versuchsreihe mit Salicylsäure ausgeführt, weil diese Substanz, Menschen und Säugetieren verfüttert, bekanntlich eine erhebliche Steigerung der Harnsäureausscheidung verursacht; es ergab sich, daß auch durch Salicylsäure die Xanthinoxydation in den Rinderleberauszügen beschleunigt wird, wenngleich in schwächerem Grade als durch Tartron- oder Dialursäure.

Die gewonnenen Resultate sind verzeichnet in Tabelle IV. Zur besseren Übersicht seien in dieser Tabelle die bei der Digestion des Auszuges ohne Salicyl-, Dialur- oder Tartronsäure erhaltenen Werte wiederholt, welche bereits in Tabelle III bei Extrakt II sub D-I angeführt wurden.

Die Versuchsbedingungen waren dieselben wie oben. Es wurden wieder für jede Probe $380 \mathrm{ccm}$ des Auszuges (entspr. $190 \mathrm{~g}$ Leberbrei) verwendet; hinzugefügt wurden zu den Proben

D, E, F je $20 \mathrm{ccm}^{1 / 2-\mathrm{n} \mathrm{Na}} \mathrm{CO}_{8}$-Lösung (s. Tab. III, Extr. II, Probe D-F),

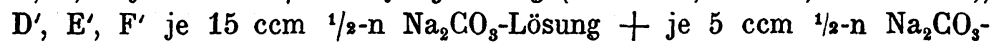

Lösung, die 0,5 g Salicylsäure enthielten,

$D^{\prime \prime}, E^{\prime \prime}, F^{\prime \prime}$ je $15 \mathrm{ccm}^{1 / 2}-\mathrm{n} \mathrm{Na}_{2} \mathrm{CO}_{8}$-Lösung + je $5 \mathrm{ccm}{ }^{1 / 2}-\mathrm{n} \mathrm{Na}_{8} \mathrm{CO}_{3}-$ Lösung, die $0,5 \mathrm{~g}$ Dialursäure enthielten,

$D^{\prime \prime \prime}, E^{\prime \prime \prime}, F^{\prime \prime \prime}$ je $15 \mathrm{ccm} \mathrm{M}^{1 / 2}-\mathrm{n} \mathrm{Na}_{8} \mathrm{CO}_{8}$-Lösung + je $5 \mathrm{ccm}{ }^{1 / 2}-\mathrm{n} \mathrm{Na}_{8} \mathrm{CO}_{3}-$ Lösung, die 0,3 g Tartronsäure enthielten; 
ferner zu den Proben

G, H, I je $20 \mathrm{ccm} \mathrm{1/2-n} \mathrm{Na}_{2} \mathrm{CO}_{3}$-Lösung mit $0,5 \mathrm{~g} \mathrm{Xanthin} \mathrm{(s.} \mathrm{Tab.} \mathrm{III,}$ Extr. II, Probe G-I),

$\mathrm{G}^{\prime}, \mathrm{H}^{\prime}, \mathrm{I}^{\prime}$ je $15 \mathrm{ccm}^{1 / 2-\mathrm{n} \mathrm{Na}} \mathrm{CO}_{8}$-Lösung mit $0,5 \mathrm{~g} \mathrm{Xanthin} \mathrm{+} \mathrm{je} 5 \mathrm{ccm}$ 1/2-n $\mathrm{Na}_{2} \mathrm{CO}_{3}$-Lösung, die 0,5 g Salicylsäure enthielten,

$G^{\prime \prime}, H^{\prime \prime}, I^{\prime \prime}$ je $15 \mathrm{ccm}^{1 / 2} / \mathrm{n} \mathrm{Na}_{2} \mathrm{CO}_{3}$-Lösung mit $0,5 \mathrm{~g}$ Xanthin + je $5 \mathrm{ccm}$ ${ }_{1 / 2-\mathrm{n} \mathrm{Na}} \mathrm{CO}_{3}$-Lösung, die $0,5 \mathrm{~g}$ Dialursäure enthielten,

$G^{\prime \prime \prime}, \mathrm{H}^{\prime \prime \prime}, \mathrm{I}^{\prime \prime \prime}$ je $15 \mathrm{ccm}{ }^{1 / 2} / \mathrm{n} \mathrm{Na}_{8} \mathrm{CO}_{3}$-Lösung mit $0,5 \mathrm{~g} \mathrm{Xanthin} \mathrm{+} \mathrm{je} 5 \mathrm{ccm}$ $1 / 2-\mathrm{n} \mathrm{Na}_{2} \mathrm{CO}_{8}$-Lösung, die $0,3 \mathrm{~g}$ Tartronsäure enthielten.

Durch sämtliche Proben wurde während der ganzen Dauer der Digestion ein lebhafter Sauerstoffstrom hindurchgeleitet.

Aus der umstehenden Tabelle ergibt sich, daß die Digestion des purinbasenarmen Auszuges mit (Salicylsäure), Dialursäure und Tartronsäure allein in allen Fällen so gut wie wirkungslos war; daß dagegen bei Anwesenheit von Xanthin in den Versuchen mit 1- oder $\left.2^{1}\right|_{\mathbf{2}}$-stündiger Digestionsdauer durch den Salicyl-, Dialur- und Tartronsäurezusatz stets eine erhebliche Steigerung der Harnsäurebildung bewirkt wurde. So lieferte der Extrakt

bei einer Digestionsdauer von

1 Stunde $21 / 2$ Stunden

mit $0,5 \mathrm{~g}$ Xanthin

$0,16 \mathrm{~g} \quad 0,25 \mathrm{~g}$

mit $0,5 \mathrm{~g}$ Xanthin $+0, \overline{\mathrm{g}} \mathrm{g}$ Dialursäure

0,22 .

0,35 .

mit $0,5 \mathrm{~g}$ Xanthin $+0,3 \mathrm{~g}$ Tartronsäure

0,29 ,

$\mathbf{0 , 3 7}$, Harnsäure.

Mit der durch die Salicyl-, Dialur- und Tartronsäure verursachten Harnsäurevermehrung geht ausnahmslos eine Verminderung des nicht oxydierten Xanthinrestes Hand in Hand (vgl. Kol. c der Tab.), und da diese Verminderung in jedem Falle den Harnsäurezuwachs an Größe übertrifft, so finden wir den Gesamtpurin-N (Kol. d) in den mit Xanthin + Salicylsäure, Dialursäure oder Tartronsäure digerierten Proben regelmäßig kleiner, als in den bloß mit Xanthin versetzten Proben.

Nach 6 stündiger Digestion ist eine Harnsäuresteigerung in der salycilsäure- und tartronsäurehaltigen Probe nicht mehr zu konstatieren, dagegen ist die Herabsetzung der Purinbasenmenge (und damit des Gesamtpurin-N) auch dann noch deutlich ausgesprochen, sodaß man hierin wohl die sicherste und konstanteste Wirkung der gewählten Zusätze erblicken muß. 
Tabelle IV.

\begin{tabular}{|c|c|c|c|c|c|c|}
\hline Probe & Zusatz & $\begin{array}{c}\text { Di- } \\
\text { gestions- } \\
\text { dauer in } \\
\text { Stunden }\end{array}$ & \begin{tabular}{|c|}
$\mathbf{a}$ \\
Harnsäure \\
$\mathbf{m g}$
\end{tabular} & $\begin{array}{c}\text { b } \\
\text { Harn- } \\
\text { säure-N } \\
\text { mg }\end{array}$ & $\begin{array}{c}\text { c } \\
\text { Purin- } \\
\text { basen-N } \\
\text { mg }\end{array}$ & $\begin{array}{c}\text { d } \\
\text { Gesamt- } \\
\text { purin-N } \\
\text { mg }\end{array}$ \\
\hline D & \multirow{3}{*}{$\varnothing$} & 1 & 3,0 & 1,0 & Spuren & 1,0 \\
\hline $\mathrm{E}$ & & 2,5 & 5,1 & 1,7 & $\varnothing$ & 1,7 \\
\hline $\mathbf{F}$ & & 6 & $\varnothing$ & $\varnothing$ & $\emptyset$ & $\varnothing$ \\
\hline $\mathrm{D}^{\prime}$ & \multirow{3}{*}{$\begin{array}{c}\text { Je } 500 \text { mg } \\
\text { Salicyl- } \\
\text { säure }\end{array}$} & 1 & 3,7 & 1,2 & Spuren & 1,2 \\
\hline$E^{\prime}$ & & 2,5 & 4,8 & 1,6 & $\emptyset$ & 1,6 \\
\hline$F^{\prime}$ & & 6 & 4,3 & 1,4 & $\varnothing$ & 1,4 \\
\hline $\mathrm{D}^{\prime \prime}$ & \multirow{3}{*}{$\begin{array}{l}\text { Je } 500 \mathrm{mg} \\
\text { Dialur- } \\
\text { säure }\end{array}$} & 1 & 4,8 & 1,6 & Spuren & 1,6 \\
\hline$E^{\prime \prime}$ & & $2, \check{0}$ & 5,3 & 1,8 & $\underset{\text { Spuren }}{\operatorname{minimalste}}$ & 1,8 \\
\hline $\mathrm{F}^{\prime \prime}$ & & 6 & 4,9 & 1,6 & $\varnothing$ & 1,6 \\
\hline $\mathrm{D}^{\prime \prime \prime}$ & \multirow{3}{*}{$\begin{array}{c}\text { Je } 500 \mathrm{mg} \\
\text { Tartron- } \\
\text { säure }\end{array}$} & 1 & 4,3 & 1,4 & 2,2 & 3,6 \\
\hline$E^{\prime \prime \prime}$ & & 2,5 & 5,3 & 1,8 & $\varnothing$ & 1,8 \\
\hline$F^{\prime \prime \prime}$ & & 6 & 4,6 & 1,5 & $\varnothing$ & 1,5 \\
\hline $\mathrm{G}$ & \multirow{3}{*}{$\begin{array}{c}\text { Je } 500 \mathrm{mg} \\
\text { Xanthin } \\
\text { (entsprechend } \\
184,2 \mathrm{mg} \mathrm{N})\end{array}$} & 1 & 163,5 & 54,5 & 140,6 & 195,1 \\
\hline $\mathrm{H}$ & & 2,5 & 247,1 & 82,4 & 95,0 & 177,4 \\
\hline I & & 6 & 236,0 & 78,7 & 39,4 & 118,1 \\
\hline$G^{\prime}$ & \multirow{3}{*}{$\begin{array}{c}\text { Je } 500 \mathrm{mg} \\
\text { Xanthin } \\
\text { (entsprechend } \\
184,2 \mathrm{mg} \mathrm{N} \text { ) } \\
\text { + je } 500 \mathrm{mg} \\
\text { Salic ylsäur } \\
\end{array}$} & 1 & 198,6 & 66,8 & 187,8 & 194,0 \\
\hline $\mathrm{H}^{\prime}$ & & 2,5 & 290,4 & 96,8 & 69,0 & 165,8 \\
\hline $\mathrm{I}^{\prime}$ & & 6 & 232,0 & 77,3 & 12,8 & 90,1 \\
\hline$G^{\prime \prime}$ & \multirow{3}{*}{$\begin{array}{c}\text { Je } 500 \mathrm{mg} \\
\text { Xanthin } \\
\text { (entsprechend } \\
18+, 2 \mathrm{mg} \mathrm{N} \\
+ \text { je } 500 \mathrm{mg} \\
\text { Dialursäure }\end{array}$} & 1 & 225,2 & 75,1 & 116,4 & 191,5 \\
\hline $\mathrm{H}^{\prime \prime}$ & & 2,5 & 352,4 & 117,5 & 53,2 & 170,7 \\
\hline$I^{\prime \prime}$ & & 6 & 275,7 & 91,9 & 8,3 & 100,2 \\
\hline$G^{\prime \prime \prime}$ & \multirow{3}{*}{$\begin{array}{c}\text { Je } 500 \mathrm{mg} \\
\text { Xanthin } \\
\text { (entsprechend } \\
184,2 \mathrm{mg} \mathrm{N} \text { ) } \\
\text { + je } 500 \mathrm{mg} \\
\text { Tartrons u u e }\end{array}$} & 1 & 290,7 & 96,9 & 90,3 & 187,2 \\
\hline $\mathrm{H}^{\prime \prime \prime}$ & & 2,5 & 368,6 & 122,9 & 18,4 & 141,3 \\
\hline$I^{\prime \prime \prime}$ & & 6 & 230,0 & 76,7 & 1,9 & 78,6 \\
\hline
\end{tabular}


Über oxydative etc. Bildung von Harnsäure in Rinderleberauszug. 521

Ein völlig klares Bild von dem Einflusse der zugesetzten Substanzen gibt uns die Konstantenberechnnng, die zu der nachfolgenden Zusammenstellung führt:

$560 \mathrm{~V} \cdot \mathbf{a}=200 \mathrm{mg} . \quad 560 \mathrm{~V} \cdot \mathbf{b}=1,5 \mathrm{mg}$.

\begin{tabular}{|c|c|c|c|c|c|c|}
\hline Versuchsreihe & Probe & $560 \mathrm{~V}(\mathrm{a}-\mathrm{x})$ & $560 \cdot y$ & $\begin{array}{c}\mathrm{t} \text { in } \\
\text { Stun- } \\
\text { den }\end{array}$ & $\mathbf{k}_{\mathbf{1}}$ & $\mathbf{k}_{2}$ \\
\hline $\begin{array}{c}3 . \\
\text { Xanthin allein (vgl. die } \\
\text { Zusammenstellung auf } \\
\text { S. 514) }\end{array}$ & $\begin{array}{l}\mathrm{G} \\
\mathrm{H} \\
\mathrm{I}\end{array}$ & $\begin{array}{r}140,6 \\
95,0 \\
39,4\end{array}$ & $\begin{array}{r}6,4 \\
24,1 \\
83,4\end{array}$ & $\begin{array}{l}1 \\
2,5 \\
6\end{array}$ & $\begin{array}{l}0,352 \\
0,300 \\
0,270\end{array}$ & $\begin{array}{l}0,210 \\
0,200 \\
0,200\end{array}$ \\
\hline \multicolumn{5}{|c|}{ Mittel: } & $\mathbf{0 , 3 0 7}$ & 0,203 \\
\hline $\begin{array}{c}\mathbf{4} . \\
\text { Xanthin }+ \text { Salicyl- } \\
\text { säure }\end{array}$ & $\begin{array}{l}\mathrm{G}^{\prime} \\
\mathrm{H}^{\prime} \\
\mathrm{I}^{\prime}\end{array}$ & $\begin{array}{r}127,8 \\
69,0 \\
12,8\end{array}$ & $\begin{array}{r}7,5 \\
35,7 \\
111,4\end{array}$ & $\begin{array}{l}1 \\
2,5 \\
6\end{array}$ & $\begin{array}{l}0,448 \\
0,426 \\
0,448\end{array}$ & $\begin{array}{l}0,200 \\
0,220 \\
0,220\end{array}$ \\
\hline \multicolumn{5}{|c|}{ Mittel: } & 0,441 & 0,213 \\
\hline $\begin{array}{c}\mathbf{5} . \\
\text { Xanthin } \\
\text { säure }\end{array}$ & $\begin{array}{l}\mathrm{G}^{\prime \prime} \\
\mathrm{H}^{\prime \prime} \\
\mathrm{I}^{\prime \prime}\end{array}$ & $\begin{array}{r}116,4 \\
53,2 \\
8,3\end{array}$ & $\begin{array}{r}10,0 \\
30,8 \\
101,3\end{array}$ & $\begin{array}{l}1 \\
2,5 \\
6\end{array}$ & $\begin{array}{l}0,541 \\
0,530 \\
0,537\end{array}$ & $\begin{array}{l}0,230 \\
0,160 \\
0,174\end{array}$ \\
\hline \multicolumn{5}{|c|}{ Mittel: } & $\mathbf{0 , 5 3 6}$ & 0,188 \\
\hline $\begin{array}{c}6 . \\
\text { Xanthin } \\
\text { săure }\end{array}$ & $\begin{array}{l}G^{\prime \prime \prime} \\
H^{\prime \prime \prime} \\
I^{\prime \prime \prime}\end{array}$ & $\begin{array}{r}90,3 \\
18,4 \\
1,9\end{array}$ & $\begin{array}{r}14,3 \\
60,2 \\
122,9\end{array}$ & $\begin{array}{l}1 \\
2,5 \\
6\end{array}$ & $\begin{array}{l}0,794 \\
0,953 \\
0,776\end{array}$ & $\begin{array}{l}0,245 \\
0,250 \\
0,204\end{array}$ \\
\hline \multicolumn{5}{|c|}{ Mittel: } & 0,841 & 0,233 \\
\hline
\end{tabular}

Diese Zusammenstellung lehrt zunächst wieder, daß unsere Rechnung auf richtigen Voraussetzungen beruhen muß; denn die Werte für $k_{1}$ und $k_{2}$ zeigen in jeder einzelnen Versuchsreihe mehr oder weniger deutlich die geforderte Konstanz. Vor allem aber beweisen die obigen Zahlen ganz unzweideutig, daß die Wirkung des Salicylsäure-, Dialursäure-und Tartronsäurezusatzes in einer Beschleunigung der Xanthinoxydation $\left(k_{1}\right)$ besteht: die Geschwindigkeit der letzteren erscheint z. B. in den Tartronsäureversuchen (6. Reihe) auf mehr als das Doppelte erhöht. 
Der Umstand, daß die Harnsäur ezerstörung $\left(k_{2}\right)$ dabei so ziemlich unverändert bleibt, spricht abermals dafür, daß die Bildung und die Weiterzersetzung der Harnsäure in den Leberauszügen nicht durch ein und dasselbe Ferment bewirkt werden.

Für Zeitpunkt und Höhe des Harnsäuremaximums in jeder der obigen Versuchsreihen ergeben sich nach Gleichung (5) bei Benützung der Mittelwerte von $k_{1}$ und $k_{2}$ die nachstehenden Daten:

\begin{tabular}{c|c|c|c}
\hline \hline $\begin{array}{c}\text { Versuchs- } \\
\text { reihe }\end{array}$ & $\begin{array}{c}560 \mathrm{~V}\left(\mathbf{a}-\mathbf{x}_{\tau}\right) \\
\text { [Purinbasen-N in mg] }\end{array}$ & $\begin{array}{c}560 \mathrm{~V}\left(\mathbf{b}+\mathbf{x}_{\tau}-\mathbf{y}_{\tau}\right) \\
\text { [Harnsäure-N in mg] }\end{array}$ & $\begin{array}{c}\tau \\
\text { in Stunden }\end{array}$ \\
\hline 3 & 58,3 & 90,4 & 3,98 \\
4 & 49,6 & 101,7 & 3,16 \\
5 & 39,9 & 117,6 & 3,04 \\
6 & 34,5 & 115,5 & 1,97
\end{tabular}

Die Wirkung der Zusätze kommt auch in diesen Zahlen klar zum Ausdruck, am deutlichsten in den Zahlen für $\tau$ : während nämlich das Harnsäuremaximum in den Versuchen mit bloßem Xanthin erst nach ca. 4 Stunden erreicht wurde, trat es bei Gegenwart von Dialursäure nach ca. 3 und bei solcher von Tartronsäure sogar schon nach ca. 2 Stunden ein.

Die graphische Darstellung der Versuchsresultate liefert uns folgende Kurven, welche in der bereits oben besprochenen Weise konstruiert sind. (Das schon auf S. 516 verzeichnete Kurvenpaar für die 3. Versuchsreihe ist hier nicht wiederholt.)

Ein Vergleich des Kurvenbildes der 6. Versuchsreihe mit jenem der 3. Versuchsreihe (S. 516) läßt die durch die Tartronsäure verursachte Beschleunigung der Reaktion ohne weiteres erkennen.

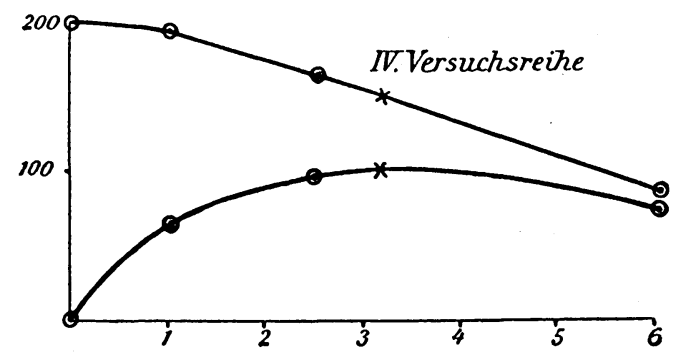



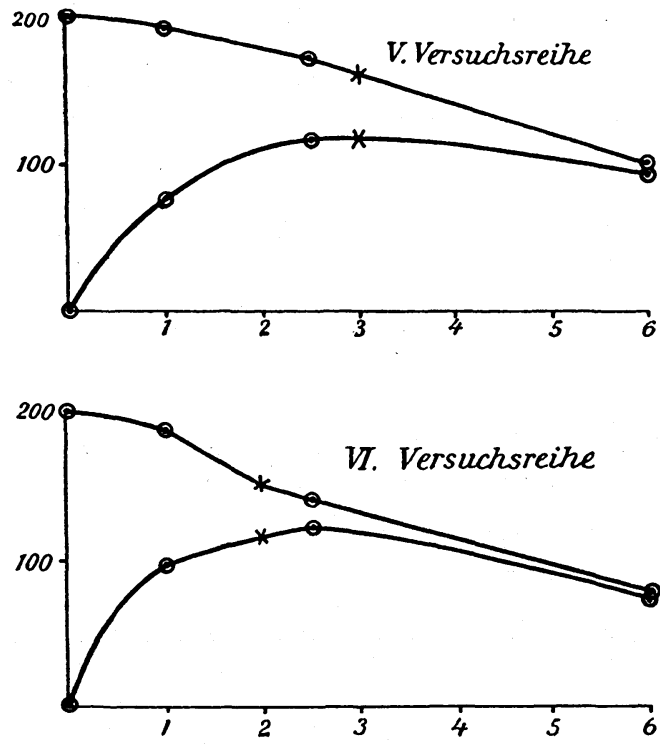

Es erhebt sich nun die Frage: Ist der Einfluß der Salicyl-, Dialur- und Tartronsäure auf eine spezifische Wirkung dieser Substanzen oder aber auf die mit dem Zusatz verbundene Alkaleszenzabnahme zurückzuführen? Zur Orientierung hierüber wurden mit ein und demselben Extrakte zwei Versuchsreihen bei verschiedenen Alkaleszenzgraden angestellt.

Es kam hier ein purinbasenarmer Rinderleberauszug zur Anwendung, durch welchen nach seiner Herstellung zunächst mehrere Stunden lang bei $40^{\circ} \mathrm{C}$ ein sehr kräftiger Luftstrom hindurchgeleitet worden war. Hierdurch wurde die geringfügige in dem Auszuge vorhandene Purinbasenmenge großenteils in Harnsäure übergeführt, sodaß der Extrakt an Basen noch ärmer, an Harnsäure aber etwas reicher erscheint, als die übrigen in meinen Versuchen benützten Extrakte.

Für jede Probe wurden $400 \mathrm{ccm}$ Auszug (entsprechend ca. $200 \mathrm{~g}$ Leberbrei) verwendet; hinzugefügt wurden zu den Proben

$\mathrm{B}, \mathrm{C}, \mathrm{D}\}$ je $25 \mathrm{ccm} 1 / 4 \mathrm{n}-\mathrm{Na}_{2} \mathrm{CO}_{8^{-}}+\left\{\right.$je $75 \mathrm{ccm} 1 / 4-\mathrm{n} \mathrm{Na}_{8} \mathrm{CO}_{3}-\mathrm{Lösung}$

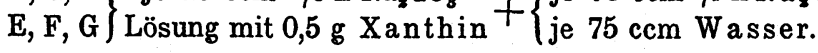

Die Flüssigkeit war somit in den Proben B, C, D 1/80-normal, in den Proben E, F, G 1/80-normal in bezug auf Natriumkarbonat; dabei besaß aber die Fermentkonzentration in allen Fällen denselben Wert, da der Extrakt für jede Probe in gleicher Weise auf $5 / 4$ verdünnt worden war. 
Über die Resultate berichtet

Tabelle V.

\begin{tabular}{|c|c|c|c|c|c|c|c|c|}
\hline Probe & $\begin{array}{c}\text { Alkaleszenz- } \\
\text { grad }\end{array}$ & Zusatz & $\begin{array}{l}\mathrm{O}_{2-} \\
\text { Darch- } \\
\text { loitang }\end{array}$ & $\begin{array}{l}\text { Diges- } \\
\text { tions- } \\
\text { dauer }\end{array}$ & $\begin{array}{c}\text { a } \\
\text { Harn- } \\
\text { säure } \\
\text { mg }\end{array}$ & $\begin{array}{c}\text { b } \\
\text { Harn- } \\
\text { säure- } \\
\text { N } \\
\text { mg }\end{array}$ & $\begin{array}{c}\text { c } \\
\text { Purin- } \\
\text { basen- } \\
\mathbf{N} \\
\mathbf{m g}\end{array}$ & $\begin{array}{c}\mathbf{d} \\
\text { Gesamt- } \\
\text { purin-N } \\
\text { mg }\end{array}$ \\
\hline A & - & $\varnothing$ & - & $\varnothing$ & 59,5 & 19,8 & 9,1 & 28,9 \\
\hline B & $1 / 20-n$ & $\mathrm{Je} 500 \mathrm{mg}$ & + & 2 & 379,8 & 126,6 & 61,9 & 188,5 \\
\hline $\mathbf{G}$ & in bezug auf & Xanthin & + & 4 & 375,6 & 125,2 & 24,4 & 149,6 \\
\hline D & $\mathrm{Na}_{2} \mathrm{CO}_{8}$ & & + & 6 & 316,8 & 105,6 & 8,2 & 113,8 \\
\hline E & $1 / 80-n$ & sprechend & + & 2 & 290,2 & 96,7 & 109,2 & 205,9 \\
\hline $\mathbf{F}$ & in bezug auf & 184,2 & + & 4 & 381,6 & 127,2 & 66,5 & 193,7 \\
\hline G & $\mathrm{Na}_{8} \mathrm{CO}_{3}$ & $\mathrm{mg} \mathrm{N}$ ) & + & 6 & 426,4 & 142,1 & 35,8 & 177,9 \\
\hline
\end{tabular}

Bemerkung: 0,2821 $\mathrm{g}$ der bei Probe B erhaltenen Harnsäure zur $\mathrm{N}$-Bestimmung verwendet: verbraucht $67,32 \mathrm{ccm}{ }^{1 / 10 \mathrm{n}}$-Säure, entsprechend $0,09425 \mathrm{~g} \mathrm{~N}$ : gef. : $\mathbf{3 3}, \mathbf{4 1} \% \mathrm{~N}$.

In welchem Sinne und Grade hier die Xanthinoxydation und die Harnsäurezerstörung durch den Alkaleszenzgrad beeinflußt wurde, darüber gibt die Konstantenberechnung Autschluß:

\begin{tabular}{|c|c|c|c|c|c|c|}
\hline Versuchsreihe & Probe & $560 \mathrm{~V}(\mathrm{a}-\mathbf{x})$ & $560 \mathrm{~V} \cdot \boldsymbol{y}$ & $\begin{array}{l}t \text { in } \\
\text { Stun- } \\
\text { den }\end{array}$ & $\mathbf{k}_{\mathbf{1}}$ & $\mathbf{k}_{\mathbf{2}}$ \\
\hline \multirow{3}{*}{$\begin{array}{c}7 . \\
\left(1 / 80-\mathrm{n} \mathrm{Na} \mathrm{CO}_{3}\right)\end{array}$} & B & 61,9 & 24,6 & 2 & 0,569 & 0,139 \\
\hline & G & 24,4 & 63,5 & 4 & 0,517 & 0,150 \\
\hline & D & 8,2 & 99,3 & 6 & 0,527 & 0,149 \\
\hline ? & \multicolumn{4}{|c|}{ Mittel: } & 0,538 & 0,146 \\
\hline \multirow{3}{*}{$\begin{array}{c}8 . \\
\left(1 / 80-n \mathrm{Na}_{2} \mathrm{CO}_{3}\right)\end{array}$} & $\mathbf{E}$ & 109,2 & 7,2 & 2 & 0,286 & 0,058 \\
\hline & $\mathbf{F}$ & 66,5 & 19,4 & 4 & 0,267 & 0,056 \\
\hline & G & 35,8 & 35,2 & 6 & 0,281 & 0,056 \\
\hline \multicolumn{5}{|c|}{ Mittel: } & 0,278 & 0,057 \\
\hline
\end{tabular}


Über oxydative etc. Bildung von Harnsäure in Rinderleberauszug. 525

Die geringere Alkaleszenz, die in den Proben der 8. Versuchsreihe herrschte, bewirkte also eine beträchtliche Herabsetzung der Werte sowohl von $\mathbf{k}_{1}$, wie auch von $\mathbf{k}_{2}$, durch welche das Harnsäuremaximum, wie die nachstehenden Zahlen lehren, von der 3. bis zur 7. Stunde hinausgerückt wurde.

\begin{tabular}{c|c|c|c}
\hline $\begin{array}{c}\text { Versuchs- } \\
\text { reihe }\end{array}$ & $\begin{array}{c}560 \mathrm{~V}\left(\mathbf{a}-\mathbf{x}_{\tau}\right) \\
\text { [Purinbasen-N in mg] }\end{array}$ & $\begin{array}{c}560 \mathrm{~V}\left(\mathbf{b}+\mathbf{x}_{\boldsymbol{\tau}}-\mathbf{y}_{\tau}\right) \\
\text { [Harnsäure-N in mg] }\end{array}$ & $\begin{array}{c}\tau \\
\text { in Stunden }\end{array}$ \\
\hline 7 & 35,6 & 130,9 & 3,16 \\
8 & 28,8 & 141,9 & 6,93
\end{tabular}
ausgeführt.

Die Berechnung ist wieder mit den Mittelwerten von $k_{1}$ und $k_{2}$

Dies Verhalten prägt sich denn auch in den zugehörigen Kurvenbildern mit aller Deutlichkeit aus.
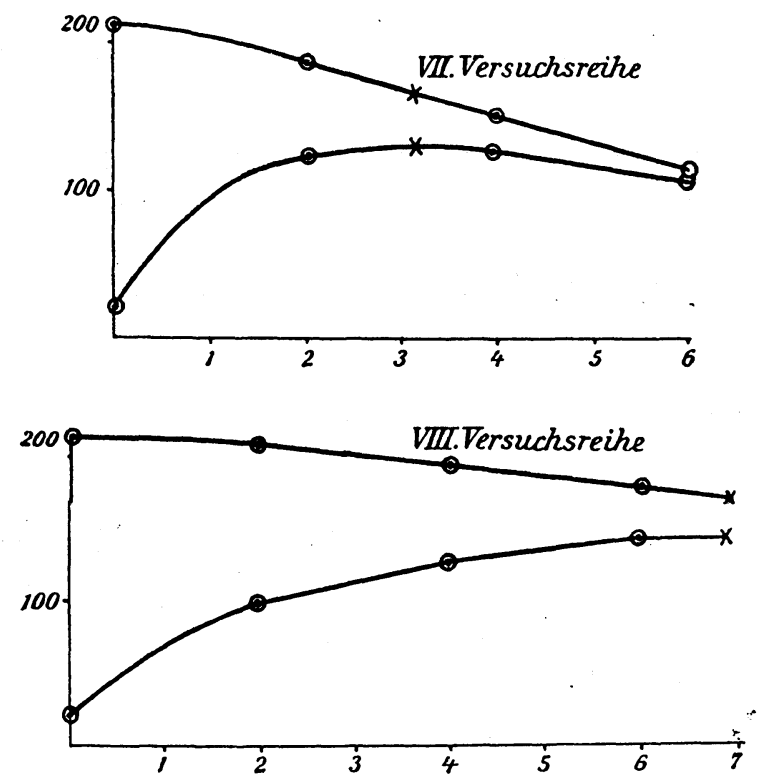

Da somit Alkaleszenzverminderung innerhalb der für die 3.-6. Versuchsreihe in Betracht zu ziehenden Grenzen eine Verzögerung der Xanthinoxydation zur Folge hatte, so kann die Beschleunigung der letzteren bei Dialur- oder Tartron-

Hoppe-Seyler's Zeitschrift f. physiol. Chemie. XLIII. 
säurezusatz wohl kaum auf der hierdurch bedingten Alkaleszenzabnahme beruhen. Wahrscheinlich liegt vielmehr eine spezifische Wirkung der genannten Stoffe vor, die in den obigen Versuchen den Einfluß der gleichzeitigen Alkaleszenzverringerung sogar erheblich überkompensiert hat.

Wie dem übrigens auch sei, soviel steht jedenfalls außer Zweifel, daß sich die Dialursäure und die Tartronsäure in meinen Versuchen nicht - im Sinne der von Wiener vertretenen Ansicht - als Ausgangsmaterialien für eine synthetische Harnsäurebildung erwiesen haben, sondern daß sie lediglich durch Beschleunigung der Purinbasenoxydation, ähnlich wie Salicylsäure, eine Steigerung der von dem Leberauszuge gelieferten Harnsäuremenge bewirkten. Mit dieser Erscheinung hatte es offenbar auch Wiener zu tun, da er mit gewöhnlichen, d. h. zweifellos stark purinbas enhaltigen Rinderleberextrakten arbeitete.

\section{Résumé.}

Zum Schlusse seien die gewonnenen Resultate noch einmal zusammengefaßt und die Folgerungen hinzugefügt, die sich aus ihnen für die Entstehungsweise der Säugetierharnsäure ergeben.

Die beschriebenen Versuche bestätigen zunächst die bekannten älteren Angaben, nach welchen Rinderleberauszug zugesetztes Xanthin und Hypoxanthin durch Vermittelung eines Enzyms, der Xanthinoxydase, in Harnsäure überzuführen vermag. Sie lehren ferner, daß auch die «spontane», d. h. ohne Xanthinkörperzusatz erfolgende Harnsäurebildung, die bei der Digestion gewöhnlicher, in der Wärme hergestellter Leberextrakte mit Sauerstoff stattfindet, einzig und allein durch die enzymatische Oxydation der in den Extrakten anwesenden (freien und gebundenen) Purinbasen zustandekommt. Denn purinbasenarme Auszüge, wie man sie unter Eiskühlung leicht bereiten kann, liefern ohne Xanthinkörperzusatz bei der Digestion mit Sauerstoff so gut wie keine Harnsäure.-Außer dem Vermögen der oxydativen Harnsäure bildung 
Uber oxydative etc. Bildung von Harnsäure in Rinderleberauszug. 527

besitzen die Rinderleberextrakte - allerdings in relativ bescheidenem Ausmaße - auch die Fähigkeit, die entstandene Harnsäure wieder zu zersetzen.

Der Ablauf der zwei genannten Prozesse läßt sich bei Digestion purinbasenarmer Leberauszüge mit Xanthin messend verfolgen: beide Vorgänge sind vollständig verlaufende Reaktionen erster Ordnung. Hieraus ergeben sich für die enzymatische Oxydation der Purinbasen zwei nicht unwichtige Folgerungen, nämlich :

1. daß die Xanthinoxydase bei der Reaktion nicht merklich verbraucht wird - sonst müßten die Geschwindigkeitskoeffizienten, in denen ja die Oxydasekonzentration enthalten ist (vgl. S. 509), einen Gang aufweisen; ${ }^{1}$ )

2. daß die rückläufige Reaktion - Reduktion der Harnsäure zu Purinbasen - nicht in merklichem Maße vor sich geht.2)

Das Zusammenwirken von oxydativer Harnsäurebildung und -zersetzung verleiht der Kurve der jeweils vorhandenen Harnsäuremengen in den mit Rinderleberauszug durchgeführten Xanthinoxydationsversuchen einen ganz charakteristischen Verlauf. Die Kurve steigt an, solange die Xanthinkonzentration so groß ist, daß der Harnsäurezuwachs in jedem Zeitteilchen den Harnsäureverlust übertrifft; sie erreicht ihr Maximum, wenn die Geschwindigkeiten beider Prozesse gleich geworden sind, und sinkt sodann wieder ab, weil die Harnsäurebildung wegen des steten Kleinerwerdens der Xanthinkonzentration immer mehr und mehr hinter der Harnsäurezerstōrung zurückbleibt. Zeitpunkt und Höhe des Harnsäuremaximums lassen sich für jede einzelne Versuchsreihe aus den Anfangskonzentrationen und den experimentell ermittelten Geschwindigkeits-

1) Für ein anderes Enzym, das Labferment, haben kürzlich Reichel und Spiro, Hofmeisters Beitr., Bd. 6, S. 68, auf ganz anderem Wege den gleichen Nachweis erbracht.

2) Diese Tatsache ist deshalb besonders hervorzuheben, weil Kutscher und Seemann kürzlich (Zentralbl. f. Physiol., Bd. 17, S. 715) die gänzlich unbegründete Vermutung ausgesprochen haben, die Harnsäure könne im Säugetierkörper zu Purinbasen reduziert werden! 
konstanten nach einer auf S. 511 angegebenen Formel berechnen. Die Formel dürfte auch in anderen Fällen bei der Untersuchung von Reaktionen mit Folgewirkung gute Dienste leisten.

Der Geschwindigkeitskoeffizient der Harnsäure zer set zung $\mathrm{k}_{2}$ wird nicht durch alle Einwirkungen in dem gleichen Sinne und Grade beeinflußt, wie jener der Harnsäurebildung $\mathrm{k}_{1}$ (vgl. S. 514 und 522); offenbar werden somit die beiden Vorgänge im Leberauszug durch verschiedene Fermente ausgelöst. Dies ergibt sich übrigens schon daraus, daß die Xanthinoxydase auch in Organen $\mathrm{zu}$ finden ist, denen das Harnsäurezerstörungsvermögen vollständig abgeht, z. B. in der Milz. ${ }^{1}$ ) Die Xanthinoxydase muß hiernach die Eigentümlichkeit besitzen, zwar das schwer oxydable Xanthin, resp. Hypoxanthin, $\left.{ }^{2}\right)$ nicht aber die durch gewöhnliche Oxydationsmittel so leicht zerstörbare Harnsäure anzugreifen, während sie also befähigt ist, die zyklische Amidinbindung durch Oxydation in zyklische Harnstoffbindung überzuführen (vgl. die unten stehenden Formeln), so vermag sie augenscheinlich nicht, an die Kohlenstoffatome 4 und 5 des Purinkernes unter Lösung der Doppel-

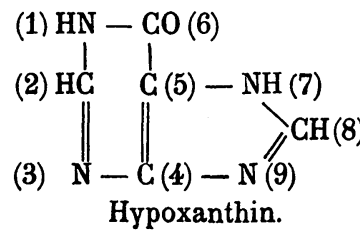<smiles>[Y4][C@H]1NC[C@H]2NC[C@H]1N2</smiles><smiles>O=C1NCC2NC(=O)NC2N1</smiles><smiles>O=C1N[C@@H](C(=O)O)NC(=O)[C@@H](O)N1</smiles>

Gemeinsame Vorstufe der Uroxansäure und des Allantoins.

1) Bei Versuchen mit Milzextrakt haben Spitzer und Schittenhelm, wie bereits erwähnt, in einigen Fällen die zugesetzten Purinbasen quantitativ als Harnsäure wiedergewonnen.

2) Guanin und Adenin sind, wie schon eingangs bemerkt, erst nach vorausgehender Desamidierung der Einwirkung der Xanthinoxydase zugänglich (Schittenhelm). 
Über oxydative etc. Bildung von Harnsäure in Rinderleberauszug. 529

bindung Sauerstoff anzulagern, wie dies bei der 0xydation von Harnsäure zu Uroxansäure oder Allantoin erforderlich ist. ${ }^{1}$ ) Man darf vielleicht vermuten, daß die Xanthinoxydase ein ganz allgemein auf (zyklische?) Amidine abgestimmtes 0xydationsferment sei; eine experimentelle Prüfung dieser Vermutung behalte ich mir vor.

Es ist selbstverständlich, daß die Xanthinoxydase nicht nur in den Organauszügen, sondern auch im intakten Säugetierkörper stets wirksam werden muß, sobald Purinbasen in die oxydasehaltigen Organe gelangen. Tatsächlich führt, wie bekannt, Verfütterung von Purinbasen und Nucleoproteiden (genügende Resorption vorausgesetzt) bei Menschen und Säugetieren ausnahmslos zu einer Steigerung der Harnsäureausscheidung. ${ }^{2}$ ) Nach den Resultaten der Organextraktversuche muß diese Steigerung zweifellos auf die wahrscheinlich größtenteils schon in der Leber stattfindende enzymatische 0xydation der aufgenommenen Purinbasen bezogen werden. Auch aus den quantitativen Verhältnissen ergibt sich übrigens ganz eindeutig die Herkunft der mehrausgeschiedenen Harnsäure aus den einverleibten Purinbasen. ${ }^{3}$ )

Daß der Säugetierkörper Purinbasen zu Harnsäure zu oxydieren vermag, ist also nicht, wie manchmal behauptet wird, eine Hypothese, sondern eine durch die Organextraktversuche und die Purinbasenfütterungsexperimente so sicher wie nur

1) Vgl. Sundwick, Diese Zeitschrift, Bd. XLI, S. 343; Behrend, Liebigs Annalen, Bd.333, S.141; vgl. insbesondere die Formeln auf S.114.

2) Dies wurde gezeigt:

Für das Hypoxanthin von Minkowski, Archiv f. exp. Pathol. und Pharm., Bd. 41, S. 403; Burian u. Schur, Pflügers Arch., Bd. 80, S. 315; Krüger und Schmid, Diese Zeitschrift, Bd. XXXIV, S. 549; Walker Hall, Purinbodies of foodstuffs, 49 (2. Aufl., S. 67);

Für das Xanthin von Burian und Schur, Pflügers Archiv, Bd. 87, S. 328 ;

Für das Guanin und das Adenin von Krüger und Schmid, 1. c., S. 563 .

Für Nucleinsäuren und Nucleoproteide von Horbaczewski, 1. c., S. 233; 0. Loewi, Arch. f. exp. Path. u. Pharm., Bd. 45, S. 160, und verschiedenen anderen.

s) Vgl. Burian u. Schur, Pflügers Archiv, Bd. 94, S. 295-310. 
irgend ein Ergebnis der Physiologie festgestellte Tatsache. Wenn neuerdings ein dem Gebiete gänzlich fernstehender Autor ${ }^{1}$ ) die zum Beweise dieser Tatsache benützten Mittel als «unzulänglich» bezeichnet und das Bestreben, die Purinbasen «mit der ihnen chemisch nahestehenden Harnsäure in Beziehung zu setzen", als ein verfehltes hinstellt, so zeugt dies eben bloß von völligem Mangel an Sachkenntnis.

Anders steht es um die von verschiedenen Seiten angenommene synthetische Bildung von Harnsäure im Säugetierorganismus. Nach den oben mitgeteilten Resultaten läßt sich in den Rinderleberauszügen eine synthetische Harnsäurebildunng neben der oxydativen auf keinerlei Weise beobachten. Sind in den digerierten Proben Purinbasen nicht oder nur in sehr kleiner Menge zugegen, so ist auch die Harnsäurebildung gleich Null oder doch äußerst geringfügig; andererseits verläuft bei Purinbasenzusatz die Harnsäurebildung solcherart, daß für sie Geschwindigkeitsgleichungen gelten, in welchen bloß Purinbasen als einzige Harnsäurequelle in Rechnung gesetzt sind. Die von Wiener als Ausgangsmaterialien für eine Harnsäuresynthese betrachteten Stoffe: Tartronsäure und Dialursäure, bewirken bei Abwesenheit von Purinbasen keine merkliche Harnsäurebildung in den Leberauszügen; bei Gegenwart von Purinbasen rufen sie allerdings die von Wiener beobachtete und auf eine Synthese bezogene Steigerung der Harnsäureproduktion hervor; aber diese Erscheinung beruht nachweislich nur auf einer durch den Zusatz der genannten Stoffe bedingten Beschleunigung der enzymatischen Purinbasenoxydation.

Hiermit fällt aber die einzige experimentelle $\mathrm{Be}$ gründung, die für die Annahme einer partiellen synthetischen Entstehung der Säugetierharnsäure bisher beigebracht worden ist. Denn die von Kossel und Steudel'2) geäußerte Vermutung, daß gewisse Pyrimidinderivate im Säugetierkörper zum synthetischen Aufbaue von Harnsäure dienen könnten, stützt

1) Gohnheim, Chemie der Eiweißkörper, 2. Aufl., S. 212.

2) Diese Zeitschrift, Bd. XXXVIII, S. 58; Bd. XXXIX, S. 136. 
Über oxydative etc. Bildung von Harnsäure in Rinderleberauszug. 531 sich bis jetzt bloß darauf, daß ein solcher Vorgang chemisch nicht undenkbar wäre.

Daß wirklich nicht nur die aus den Purinbasen der Nahrung hervorgehende exogene, sondern auch die endogene Harnsäure des Menschen und der Säugetiere auf oxydativem Wege entsteht, und zwar hauptsächlich aus dem Hypoxanthin des Muskels, das glaube ich in der nachfolgenden Mitteilung zeigen zu können. 